ZEIN MOHAMED SAMMOUR

\title{
Distúrbios miccionais em pacientes com doença de Parkinson : associação entre parâmetros clínicos e urodinâmicos
}

Tese apresentada à Faculdade de Medicina da Universidade de São Paulo para obtenção do título de Doutor em Ciências

Área de Concentração: Urologia

Orientador: Dr. Cristiano Mendes Gomes

São Paulo

2007 


\section{Dados Internacionais de Catalogação na Publicação (CIP)}

Preparada pela Biblioteca da

Faculdade de Medicina da Universidade de São Paulo

Creprodução autorizada pelo autor

\section{Sammour, Zein Mohamed}

Distúrbios miccionais em pacientes com doença de Parkinson : associação entre parâmetros clínicos e urodinâmicos / Zein Mohamed Sammour. -- São Paulo, 2007.

Tese(doutorado)--Faculdade de Medicina da Universidade de São Paulo.

Departamento de Cirurgia.

Área de concentração: Urologia.

Orientador: Cristiano Mendes Gomes.

Descritores: 1.Doença de Parkinson 2.Prevalência 3.Transtornos urinários 4.Miç̧ão 5.Qualidade de vida 6.Urodinâmica 7.Bexiga neurogênica

USP/FM/SBD-110/07 
DEDICATÓRIA 
Aos pacientes com doença de Parkinson e seus familiares, pessoas sensíveis que no seu íntimo procuram uma perspectiva mais ampla para o tratamento dessa condição.

À minha mãe, Maria Marques Sammour, e ao meu falecido pai, Mohamed Zein Abedine Sammour, que são os meus maiores motivos de orgulho e gratidão. Ela, pela generosidade, simplicidade e sabedoria. Ele, pela força e coragem.

À minha irmã, Líbia Mohamed Sammour, pela presença constante, boa vontade e dedicação. 
Aos meus irmãos Nasser José Marques Sammour e Siria Maria Sammour, a quem sou grato pela solidariedade.

Aos meus tios Antonio Pereira Marques, João Eustaquio Pereira Marques e José Pereira Marques, que sempre me apoiaram para que eu melhorasse a cada dia.

À minha esposa, Simone Nascimento Fagundes Sammour, que me faz muito feliz, e em todos os momentos consegue conciliar equilíbrio, dedicação e alegria com o nosso amor. 
AGRADECIMENTOS 
Agradeço primeiramente ao Prof. Miguel Srougi, pela valiosa oportunidade de poder realizar este trabalho.

Ao meu orientador, Dr. Cristiano Mendes Gomes, que sempre me ensinou e incentivou. Uma pessoa muito especial e que significa muito para $\operatorname{mim}$

Aos professores Egberto Reis Barbosa, Flavio Eduardo Trigo Rocha, Homero Bruschini, Nelson Íleo Dias Montelato e Sami Arap, a quem manifesto a minha gratidão e admiração.

Ao Dr. Flavio Salem Seckeff, Dr. Marcos Dall' Oglio e Dr. Roberto Iglesias Lopes, que foram fundamentais pela cooperação, participação e sugestões neste estudo.

Ao Dr. Carlos Alberto Ricetto Sacomani, Dr. Elias Assad Chedid Neto, Dr. José Bessa Jr., Dr. Ricardo Luis Vita Nunes e Dr. Ricardo Jordão Duarte, o meu sincero agradecimento pelo convívio e amizade. 
SUMÁRIO 


\section{SUMÁRIO}

Lista de Abreviaturas e Símbolos

Lista de Figuras

Lista de Tabelas

Resumo

Summary

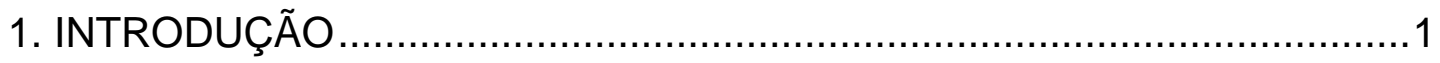

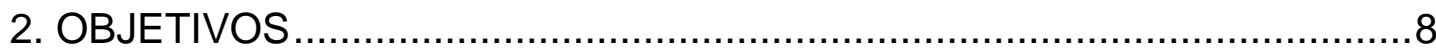

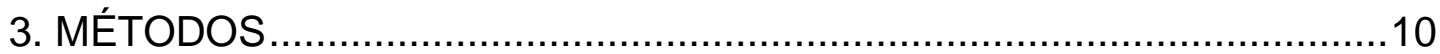

3.1 Comprometimento neurológico devido à doença de Parkinson .........12

3.2 Sintomas do trato urinário inferior em pacientes com doença de Parkinson

3.3 Associação entre sintomas do trato urinário inferior e doença de Parkinson

3.4 Impacto dos sintomas do trato urinário inferior e do comprometimento neurológico na qualidade de vida (devido a sintomas urinários)

3.5 Pacientes sintomáticos....................................................... 14

3.6 Associação entre alterações urodinâmicas e comprometimento neurológico

3.7 Sintomas urinários e qualidade de vida em homens e mulheres com disfunção miccional e doença de Parkinson............................16

3.8 Análise estatística ......................................................... 16

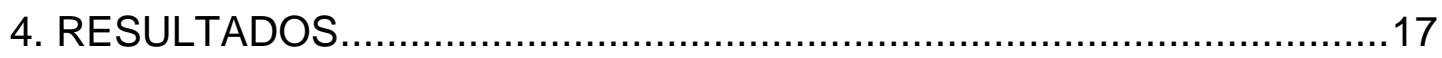

4.1 Comprometimento neurológico devido à doença de Parkinson .........18

4.2 Sintomas do trato urinário inferior em pacientes com doença de Parkinson

4.3 Associação entre sintomas do trato urinário inferior e doença de Parkinson

4.4 Impacto dos sintomas do trato urinário inferior e do comprometimento neurológico na qualidade de vida (devido a sintomas urinários)

4.5 Pacientes sintomáticos.

4.6 Associação entre anormalidades urodinâmicas e comprometimento neurológico 
4.7 Sintomas do trato urinário inferior e qualidade de vida em homens

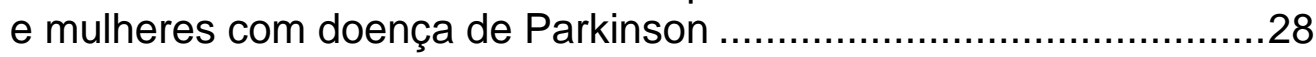

5. DISCUSSÃO

6. CONCLUSÕES

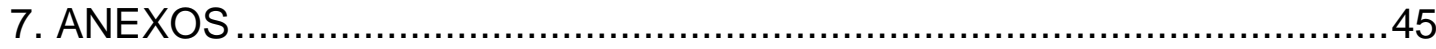

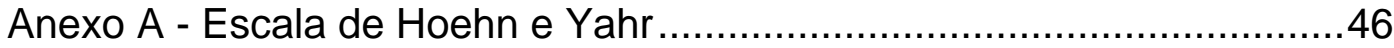

Anexo B - Escala de classificação unificada da doença de Parkinson......47

Anexo C - Questionário simplificado da Sociedade Internacional de

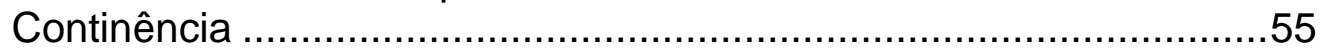

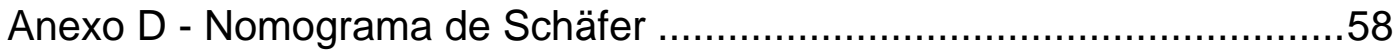

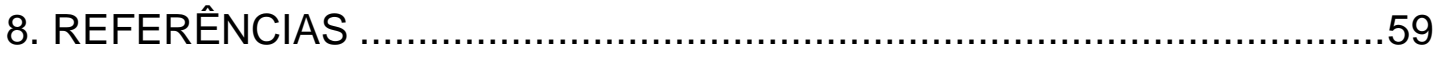

9. APÊNDICES

Apêndice I - Termo de consentimento livre e esclarecido Apêndice II - Diário miccional 


\section{LISTA DE ABREVIATURAS E SÍMBOLOS}

\begin{tabular}{|c|c|}
\hline CCmax & - capacidade cistométrica máxima \\
\hline $\mathrm{cmH}_{2} \mathrm{O}$ & - centímetros de água \\
\hline $\mathrm{CO}_{2}$ & - Dióxido de carbono \\
\hline DP & - desvio padrão \\
\hline DP & - Doença de Parkinson \\
\hline et al. & - e outros \\
\hline HC-FMUSP & - Hospital das Clínicas da Faculdade de Medicina da \\
\hline & Universidade de São Paulo \\
\hline HD & - Hiperatividade detrusora \\
\hline HY & - Hoehn and Yahr \\
\hline ICS & - International Continence Society \\
\hline ICSF & - International Continence Society / Frequency \\
\hline ICSI & - International Continence Society / Incontinence \\
\hline ICSmSF & - International Continence Society male Short Form \\
\hline ICSV & - International Continence Society / Voiding \\
\hline IPSS & - International Prostate Score Symptoms \\
\hline HPB & - Hiperplasia prostática benigna \\
\hline LUTS & - Lower urinary tract symptoms \\
\hline $\mathrm{mL}$ & - mililitros \\
\hline $\mathrm{mL} / \mathrm{cmH}_{2} \mathrm{O}$ & - mililitros por centímetros de água \\
\hline $\mathrm{mL} / \mathrm{s}$ & - mililitros por segundo \\
\hline$n^{0}$ & - número \\
\hline
\end{tabular}




\begin{tabular}{|c|c|}
\hline OIV & - Obstrução infravesical \\
\hline p. & - Página \\
\hline Pdet & - Pressão detrusora \\
\hline PdetQmax & - Pressão detrusora no fluxo máximo \\
\hline PSA & - Antígeno prostático específico \\
\hline QV & - Qualidade de vida \\
\hline vS. & - versus \\
\hline STUI & - Sintomas do trato urinário inferior \\
\hline UPDRS & - Unified Parkinson Disease Rating Scale \\
\hline VW & - very weak (muito fraca) \\
\hline$\%$ & - porcentagem \\
\hline$<$ & - menor que \\
\hline$>$ & - maior que \\
\hline$=$ & - igual a \\
\hline \pm & - mais ou menos \\
\hline cc & - centímetros cúbicos \\
\hline$p$ & - significância \\
\hline
\end{tabular}




\section{LISTA DE FIGURAS}

Figura 1 - Prevalência dos sintomas de enchimento............................19

Figura 2 - Prevalência dos sintomas de esvaziamento ..........................20

Figura 3 - Prevalência de urge-incontinência (episódios/dia) ...................20

Figura 4 - Freqüência urinária diurna (micções/dia) .............................21

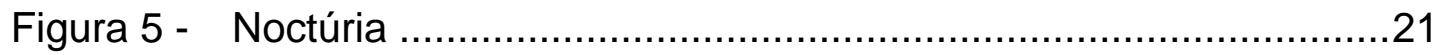

Figura 6 - Associação entre gravidade da doença de Parkinson (UPDRS) e sintomas do trato urinário inferior (ICSmSF) por

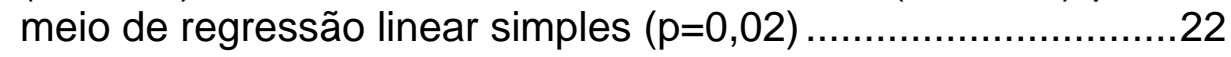

Figura 7 - Análise de correlação demonstrando efeito da gravidade da doença de Parkinson ( $\mathrm{HY}$ ) nos sintomas do trato urinário

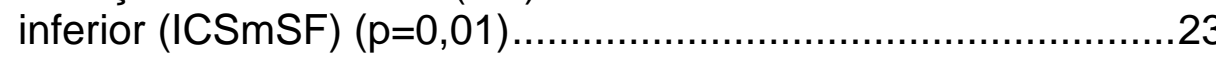




\section{LISTA DE TABELAS}

Tabela 1 - Qualidade de vida em função dos escores de sintomas

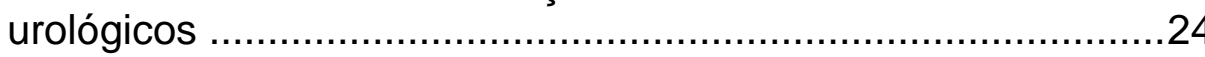

Tabela 2 - Parâmetros urodinâmicos da doença de Parkinson....................26

Tabela 3 - Achados urodinâmicos na doença de Parkinson .....................27

Tabela 4 - Associação entre achados urodinâmicos e comprometimento

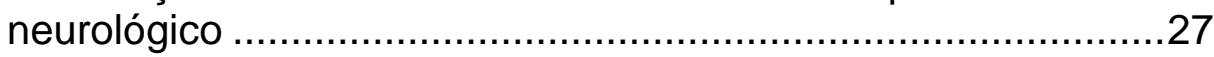

Tabela 5 - Parâmetros clínicos, sintomas do trato urinário inferior e qualidade de vida em homens e mulheres com doença de Parkinson .................................................................28 
RESUMO 
Sammour ZM. Distúrbios miccionais em pacientes com Doença de Parkinson: associação entre parâmetros clínicos e urodinâmicos [tese]. São Paulo: Faculdade de Medicina, Universidade de São Paulo; 2007. 66p.

INTRODUÇÃO E OBJETIVOS: Distúrbios miccionais são freqüentes em pacientes com doença de Parkinson, mas sua associação com parâmetros como idade do paciente, gênero, gravidade do comprometimento neurológico e duração da doença não é bem conhecida. Neste estudo, avaliamos prevalência e características dos sintomas miccionais em pacientes com doença de Parkinson e examinamos sua associação com parâmetros clínicos com potencial impacto sobre a disfunção miccional. MÉTODOS: Avaliamos prospectivamente 110 pacientes, incluindo 84 homens $(76,4 \%)$ e 26 mulheres (23,6\%), com idade média de 61,8 \pm 9,6 anos. Os critérios de inclusão foram doença de Parkinson idiopática e idade acima de 40 anos. Pacientes com outras doenças neurológicas, história de cirurgia e radioterapia pélvica foram excluídos. A duração média da doença foi 12,3 \pm 7,2 anos. O comprometimento neurológico foi avaliado pelas escalas Hoehn-Yahr (HY) e Escala Unificada de Avaliação da Doença de Parkinson (UPDRS). Os sintomas miccionais foram avaliados pelo questionário da Sociedade Internacional de Continência para Homens, incluindo uma questão de qualidade de vida relacionada a sintomas miccionais. Consideramos sintomáticos os pacientes que queriam tratamento para sua disfunção miccional. Estes foram avaliados com análise de urina, creatinina sérica, PSA, ultra-sonografia do aparelho urinário e urodinâmica. Avaliamos a associação da disfunção miccional com idade, gênero, tempo de duração da doença, grau de comprometimento neurológico, impacto na qualidade de vida e achados urodinâmicos. RESULTADOS: A avaliação neurológica demonstrou média de 3,0 \pm 0,8 na escala de HY e 70,0 \pm 31,1 na escala de UPDRS. A média do escore miccional foi de $11,9 \pm 9,3$ e os sintomas mais comuns foram noctúria em 89 $(80,9 \%)$ pacientes, urgência em $40(36,3 \%)$ e aumento da freqüência miccional em $39(35,4 \%)$ pacientes. A duração média dos sintomas miccionais foi de 3,8 $\pm 3,4$ anos. A prevalência da disfunção miccional aumentou significativamente com o aumento no grau de comprometimento neurológico, mas não com a idade nem com a duração da doença. A qualidade de vida relacionada aos sintomas miccionais foi afetada pela severidade da disfunção miccional e os sintomas de freqüência e noctúria são os de pior impacto na qualidade de vida. Sessenta e três pacientes $(57,2 \%)$ eram sintomáticos e 54 (43 homens e 11 mulheres) completaram a avaliação. Os pacientes sintomáticos apresentaram disfunção neurológica mais severa (HY médio $3,2 \pm 0,8$ vs 2,8 $\pm 0,9$ para os assintomáticos; $p=0,035)$. A idade, a duração da doença e dos sintomas miccionais não diferiram entre sintomáticos e assintomáticos. O volume médio da próstata foi $30,3 \pm 10,7$ cc. Os achados urodinâmicos foram obstrução infravesical em 38 (70,3\%) pacientes, hiperatividade detrusora em $27(50,0 \%)$, e hipocontratilidade detrusora em seis $(11,1 \%)$ pacientes. O grau de comprometimento neurológico não se associou com nenhum padrão urodinâmico. Na comparação entre homens e mulheres sintomáticos a única 
diferença observada foi a maior gravidade dos sintomas de esvaziamento nos homens. CONCLUSÕES: A maior parte dos pacientes com doença de Parkinson apresenta disfunção miccional significativa e a gravidade da doença neurológica é o principal fator preditivo para a ocorrência de disfunção miccional. Obstrução infravesical e hiperatividade detrusora são os achados urodinâmicos mais comuns, mas não estão associados com o grau de comprometimento neurológico. Homens e mulheres são similarmente afetados pelos sintomas miccionais e não demonstraram distinção quanto aos achados urodinâmicos.

Descritores: doença de Parkinson, prevalência, transtornos urinários, micção, qualidade de vida, urodinâmica, bexiga neurogênica. 
Sammour ZM. Voiding disfunction in patients with Parkinson's disease: association between clinical and urodynamic parameters [thesis]. São Paulo: "Faculdade de Medicina, Universidade de São Paulo"; 2007. 66p.

INTRODUCTION AND OBJECTIVES: Voiding dysfunction often occurs in patients with Parkinson's disease, but its association with patient's age, gender, degree of neurological impairment, and disease duration is not fully known. In this study, we assessed the prevalence and characteristics of lower urinary tract symptoms (LUTS) in patients with Parkinson's disease and examined their association with those clinical parameters that could have an impact on voiding dysfunction. METHODS: We prospectively evaluated 110 patients, of which 84 were men (76.4\%) and 26 were women (23.6\%), with a mean age of $61.8 \pm 9.6$ years. The inclusion criteria were: idiopathic Parkinson's disease and age > 40 years. Patients with other neurological diseases and history of pelvic surgery and radiotherapy were excluded. Mean duration of the disease was $12.3 \pm 7.2$ years. The neurological impairment was assessed by the Hoehn-Yahr scale (HY) and the Unified Parkinson Disease Rating Scale (UPDRS). Lower urinary tract symptoms were assessed by the International Continence Society male questionnaire, which included a question about quality of life related to LUTS. Those patients who wanted to be treated for their voiding dysfunction were considered as symptomatic. They underwent urine analysis, serum creatinine measurement, PSA, urinary tract imaging and urodynamic study. We examined the association between voiding dysfunction and age, gender, disease duration, degree of neurological impairment, impact on quality of life, and urodynamic findings. RESULTS: The neurological assessment showed a mean HY score of $3.0 \pm 0.8$ and a mean UPDRS score of $70.0 \pm 31.1$. Mean LUTS score was $11.9 \pm 9.3$, and the most prevalent symptoms were nocturia in $89(80.9 \%)$ patients, urgency in $40(36.3 \%)$, and frequency in $39(35.4 \%)$. Mean duration of LUTS was $3.8 \pm 3.4$ years. The prevalence of voiding dysfunction increased significantly with the degree of neurological impairment, but not with patient's age or disease duration. Quality of life was affected by the severity of the voiding dysfunction, and the symptoms with the worst impact on quality of life were frequency and nocturia. Sixty-three patients (57.2\%) were symptomatic and 54 (43 men and 11 women) concluded the evaluation. Symptomatic patients had more severe neurological dysfunction (mean HY $3.2 \pm 0.8$ vs $2.8 \pm 0.9$ for asymptomatic patients; $p=0.035$ ). Symptomatic and asymptomatic patients did not differ as to age, disease duration and LUTS. Mean prostate volume was $30.3 \pm 10.7 \mathrm{cc}$. The urodynamic findings were bladder outlet obstruction in 38 (70.3\%) patients, detrusor overactivity in 27 (50.0\%), and detrusor underactivity in 6 (11.1\%). The degree of neurological impairment was not associated with any urodynamic pattern. The only difference observed between symptomatic men and women was that the voiding symptoms were more severe in men. CONCLUSIONS: Most patients with Parkinson's disease have significant voiding dysfunction, and the severity of the neurological disease is the main 
predictive factor for the occurrence of voiding dysfunction. Bladder outlet obstruction and detrusor overactivity are the most common urodynamic findings, although not associated with the degree of neurological impairment. Men and women are equally affected by LUTS and show similar urodynamic findings.

Descriptors: Parkinson disease, prevalence, urination disorders, urination, quality of life, urodynamic, neurogenic bladder. 
1. INTRODUÇÃO 
A doença de Parkinson (DP) é um transtorno neurológico progressivo, com prevalência estimada de $150-200$ casos em cada 100.000 pessoas. A doença é mais prevalente entre os idosos, afetando $1 \%$ das pessoas com mais de 65 anos e $2 \%$ daquelas acima de 85 anos de idade. Moléstia de distribuição mundial (Louis et al., 2004; Samii et al., 2004; Schapira, 2004), a doença de Parkinson caracteriza-se pela degeneração dos neurônios dopaminérgicos do sistema nigroestriatal, cujo marcador é a presença de corpos de Lewy no sistema nervoso central, inclusive na substantia nigra e locus coeruleus (Samii et al., 2004).

As causas da DP são desconhecidas, porém evidências consideráveis sugerem uma etiologia multifatorial, compreendendo aspectos genéticos e ambientais. A disfunção mitocondrial, os danos oxidativos, fatores ambientais e a predisposição genética podem estar envolvidos (Hattori e Mizuno, 2004; McGeer e McGeer, 2004). Por meio de uma abordagem genético-molecular, identificaram-se cinco genes e, no mínimo, dez loci 
adicionais em formas familiares raras da DP. Desses genes, dois estão envolvidos na degradação de proteínas mediada pela ubiquitina e um terceiro gene é uma proteína chamada alfa-sinucleína expressa no terminal sináptico. Em modelos animais, observou-se que pesticidas de uso doméstico, conhecidos por conterem rotenona, causam DP. Portanto, a ação combinada de fatores genéticos e ambientais é responsável pela patogênese da DP.

Do ponto de vista clínico, a DP caracteriza-se por rigidez, tremores de repouso, bradicinesia e insuficiência autonômica. Os sintomas do trato urinário inferior (STUI) são freqüentes em pacientes com DP. Estima-se que de $27 \%$ a $78 \%$ dos pacientes apresentem distúrbios miccionais durante o curso da doença (Hattori et al., 1992; Araki e Kuno, 2000; Sakakibara et al., 2001b; Campos-Sousa et al., 2003; Winge et al., 2006). Aumento da freqüência miccional, urgência, noctúria e esvaziamento vesical incompleto são os sintomas urinários mais comuns na DP (Pavlakis et al., 1983; Araki e Kuno, 2000; Lemack et al., 2000).

A incidência dos distúrbios miccionais não parece estar relacionada ao sexo, mas, para alguns autores, os sintomas obstrutivos são mais prevalentes nos pacientes do sexo masculino (Araki et al., 2000; Lemack et al., 2000). Dois trabalhos recentemente publicados demonstram que pacientes com DP apresentam risco duas vezes maior de desenvolverem sintomas miccionais, quando comparados a grupos controle (Campos-Sousa et al., 2003; Hobson et al., 2003). 
A avaliação urodinâmica pode revelar anormalidades em até $88 \%$ dos pacientes, entre elas: hiperatividade detrusora (HD), obstrução infravesical (OIV) e hipocontratilidade detrusora (Khan et al., 1989; Junemann e Melchior, 1990; Aranda, 1993; Conquy et al., 1994; Bonnet et al., 1997). A hiperatividade detrusora corresponde a uma contração involuntária do detrusor, durante a fase de enchimento vesical, acompanhada de elevação da pressão detrusora. Pode ser responsável por um amplo espectro de sintomas, incluindo urge-incontinência, aumento da freqüência urinária, urgência miccional, noctúria e perdas urinárias insensíveis (Abrams et al., 2002). Freqüentemente está associada a doenças do sistema nervoso, como lesões suprapontinas e medulares, esclerose múltipla, doença de Alzheimer, acidente vascular cerebral e outras (Bruschini et al., 1996; Sacomani et al., 2003; Gomes et al., 2004a; Gomes et al., 2005). Na DP, a hiperatividade detrusora tem sido a alteração urodinâmica mais freqüentemente encontrada, acometendo entre 40 e $80 \%$ dos pacientes sintomáticos (Pavlakis et al., 1983; Berger et al., 1987; Winge e Fowler, 2006).

Outro achado urodinâmico habitual em pacientes com DP é a obstrução infravesical. A OIV é um distúrbio urodinâmico muito freqüente na população masculina, caracterizado por micções com pressão detrusora elevada e fluxo reduzido. Geralmente ocasiona sintomas de dificuldade miccional, como hesitação para iniciar o jato, jato fraco e intermitente, esvaziamento incompleto e necessidade de realizar esforço abdominal. Pode ser causada por problemas obstrutivos mecânicos, como aumento do volume prostático, ou por problemas funcionais, como a falta de relaxamento 
esfincteriano (Gomes et al., 2001). Mulheres também podem apresentar OIV mecânica, sendo esta geralmente causada por prolapso pélvico ou complicações de cirurgias para correção de incontinência urinária (Kuo, 2005; Mc Crery e Appel, 2006). Doenças neurológicas são a principal causa de OIV funcional, notadamente em pacientes com lesões em nível medular (Bruschini et al., 1996; Sacomani et al., 2003; Gomes et al., 2005).

Pacientes com DP podem apresentar obstrução infravesical tendo como principal causa a bradicinesia esfincteriana, ou retardo no relaxamento do esfíncter estriado. Estima-se que 30 a 50\% dos indivíduos com DP apresentem OIV (Winge e Fowler, 2006).

A correlação entre STUI e o grau de comprometimento neurológico em pacientes com DP não está bem caracterizada. O impacto da idade do paciente, a duração da DP e a gravidade do comprometimento neurológico sobre a função miccional são controversos (Araki et al., 2000; Araki e Kuno, 2000; Lemack et al., 2000; Gomes et al., 2004b).

Para alguns autores, a prevalência da disfunção miccional em pacientes com DP parece aumentar proporcionalmente à gravidade da doença (Hattori et al., 1992; Araki et al., 2000; Gomes et al., 2004b). Araki e Kuno (2000) demonstraram que os distúrbios miccionais na DP desenvolvem-se progressivamente com o avançar da doença, principalmente em indivíduos com estágios de Hoehn e Yahr (HY) igual ou superior a três.

No entanto, um recente estudo que avaliou pacientes com DP por meio de um questionário enviado por correio não encontrou correlação entre 
a idade, a duração dos sintomas e a gravidade da doença e os sintomas urinários (Hobson et al., 2003). Lemack et al. (2000) também utilizaram um questionário pelo qual avaliaram 93 pacientes com DP em estágios iniciais da doença, com HY inferior a três, e não observaram correlação entre os sintomas urinários e idade ou estágio da DP.

As diferenças entre homens e mulheres com DP em relação à disfunção miccional foram pouco abordadas na literatura médica. Como a DP acomete uma população de faixa etária mais avançada, sujeita a doenças com possível impacto sobre o trato urinário, os distúrbios miccionais podem ser multifatoriais nesses pacientes. Não seria surpresa se os homens tivessem sintomas miccionais mais intensos, notadamente em relação aos sintomas de esvaziamento e à presença de obstrução infravesical, já que podem apresentar hiperplasia benigna da próstata (Fitzpatrick, 2006). Entretanto, poucos estudos avaliaram esse assunto e colocam em dúvida se existem diferenças significativas entre homens e mulheres quanto à incidência e características dos distúrbios miccionais e anormalidades urodinâmicas.

Conforme discutido, o efeito dos parâmetros idade, gênero, gravidade da DP e tempo de duração da doença sobre os distúrbios miccionais permanece controverso na literatura médica. Da mesma forma, não é bem conhecida a associação entre esses parâmetros clínicos e urodinâmicos. A maioria dos estudos sobre disfunção miccional em pacientes com DP avaliou apenas alguns aspectos dessas complexas inter-relações, isto é, são estudos essencialmente epidemiológicos ou de achados 
urodinâmicos. Raros estudos reuniram os vários aspectos relevantes da epidemiologia e fisiopatologia dos distúrbios miccionais em pacientes com DP.

Devido à importância desta doença e da elevada prevalência e morbidade dos sintomas miccionais na população acometida, julgamos ser importante realizar um estudo abrangente dos distúrbios miccionais em pacientes com DP. 


\section{OBJETIVOS}


1. Determinar a prevalência e as características clínicas dos sintomas miccionais nos pacientes com doença de Parkinson.

2. Associar os sintomas miccionais com idade, tempo de evolução e gravidade da doença de Parkinson e avaliar o impacto dos sintomas do trato urinário inferior na qualidade de vida dos pacientes.

3. Caracterizar o distúrbio miccional por meio da avaliação urodinâmica nos pacientes sintomáticos e associar a gravidade da doença de Parkinson com as anormalidades urodinâmicas observadas.

4. Verificar se há distinção entre os sexos quanto aos sintomas urológicos, qualidade de vida e achados urodinâmicos. 


\section{MÉTODOS}


Em um estudo prospectivo com duração de três anos, avaliamos consecutivamente 110 pacientes com DP recrutados pelo ambulatório de Neurologia do Instituto Central do Hospital das Clínicas da Faculdade de Medicina da Universidade de São Paulo (HC-FMUSP), onde eram seguidos. Todos os pacientes tinham diagnóstico estabelecido de DP (Hughes et al., 1992) e foram convidados a participar do estudo, independentemente da presença de sintomas urinários. Os critérios de inclusão foram DP idiopática e idade acima de 40 anos. Os critérios de exclusão foram associações com outros transtornos neurológicos, história de cirurgia pélvica ou de próstata e radioterapia pélvica.

Este estudo foi aprovado pela Comissão de Ética do HC-FMUSP. Os pacientes concordaram em participar depois de serem minuciosamente informados sobre o objetivo do estudo e todos assinaram o termo de consentimento livre e esclarecido (Apêndice I). 
O grupo era composto por 84 homens $(76,4 \%)$ e 26 mulheres $(23,6 \%)$, com idade média de 61,8 \pm 9,6 anos (intervalo de 42 a 78 anos). A duração média da doença era de 12,3 \pm 7,2 anos (intervalo de um a 35 anos).

\subsection{Comprometimento neurológico devido à DP}

A gravidade do comprometimento neurológico foi avaliada pela escala Hoehn-Yahr (escala HY, que varia de zero a cinco) e pela Escala Unificada de Avaliação da Doença de Parkinson (escala UPDRS, que varia de zero a 147) (Hoehn e Yahr, 1967; Fahn e Elton, 1987) (Anexos A e B). A avaliação dessas escalas foi realizada por neurologistas especializados em distúrbios dos movimentos. Optamos por não avaliar os possíveis efeitos do uso de drogas anti-parkinsonianas nos STUI e nos parâmetros urodinâmicos, já que todos os pacientes usavam várias medicações pertencentes a diferentes classes de drogas anti-parkinsonianas, em diferentes dosagens, além de freqüentemente receberem outras medicações para distúrbios cognitivos e/ou psiquiátricos.

\subsection{STUI em pacientes com DP}

Os sintomas miccionais foram avaliados por meio do questionário abreviado da International Continence Society (Sociedade Internacional de Continência - ICSmSF), com pontuação que varia de zero a 54 (Donovan et 
al., 2000) (Anexo C), com 14 questões: cinco das quais sobre sintomas de esvaziamento (ICSV), seis sobre incontinência (ICSI), uma sobre freqüência, uma sobre noctúria (ICSF) e uma questão adicional sobre qualidade de vida (QV) relacionada a sintomas urinários. Solicitamos aos pacientes 0 preenchimento de um diário miccional durante três dias. Foi incluída também uma pergunta sobre a duração dos sintomas miccionais. Noctúria foi definida como a queixa de acordar para urinar ao menos uma vez durante a noite, documentada pelo diário miccional (Apêndice II).

\subsection{Associação entre STUI e DP}

Para pesquisar a influência do comprometimento neurológico sobre os sintomas urinários, os escores UPDRS e HY foram correlacionados com o ICSmSF. Além disso, avaliamos a associação dos STUI com a idade dos pacientes e com a duração da DP.

\subsection{Impacto dos STUI e do comprometimento neurológico na QV (devido a sintomas urinários)}

Associamos a QV relacionada aos sintomas urinários com a gravidade da disfunção miccional avaliada por meio do ICSmSF. Para 
determinar quais sintomas urinários exercem maior impacto sobre a QV dos pacientes com DP, avaliamos sua correlação com sintomas de esvaziamento, sintomas de incontinência e sintomas de freqüência/noctúria. Além disso, avaliamos a associação entre QV relacionada aos STUI e a gravidade da DP (UPDRS).

\subsection{Pacientes sintomáticos}

Consideramos como sintomáticos aqueles pacientes que se sentiam incomodados por sua disfunção miccional e que estavam dispostos a se submeter a tratamento, independentemente do seu escore ICSmSF. A esses pacientes foi oferecida avaliação complementar, que incluía análise do sedimento urinário, urocultura, creatinina sérica, antígeno prostático específico (PSA, somente para pacientes do sexo masculino), ultrasonografia transabdominal da próstata (pacientes do sexo masculino) e do trato urinário e estudo urodinâmico.

A avaliação urodinâmica consistiu em urofluxometria livre e determinação de resíduo pós-miccional, cistometria de enchimento e estudos de pressão/fluxo, inclusive com monitoramento eletromiográfico perineal usando eletrodos de superfície. Os parâmetros urodinâmicos analisados foram: capacidade cistométrica máxima (CCmax), complacência vesical, atividade do detrusor, taxa do fluxo máximo durante o estudo de pressão/fluxo, pressão do detrusor no fluxo máximo e urina residual. A 
hiperatividade detrusora foi definida como a contração involuntária do detrusor durante a fase de enchimento (Abrams et al., 2002). A obstrução infravesical e a contratilidade do detrusor foram avaliadas por meio do nomograma de Schäfer (Schäfer et al., 1989; Schäfer, 1990) (Anexo D). Para este estudo, pacientes com obstrução leve (áreas I e II do nomograma) foram incluídos no grupo de pacientes sem obstrução. Somente os pacientes com contratilidade do detrusor considerada como muito fraca (área VW) no nomograma foram classificados como portadores de hipocontratilidade detrusora.

Os métodos, definições e unidades estão em conformidade com os padrões recomendados pela Sociedade Internacional de Continência (Abrams et al., 2002).

\subsection{Associação entre alterações urodinâmicas e comprometimento neurológico}

Avaliamos a associação entre os achados urodinâmicos de hiperatividade detrusora e obstrução infravesical com a gravidade da DP avaliada por meio do UPDRS. 


\subsection{STUI e QV em homens e mulheres com disfunção miccional e DP}

Para avaliar a influência do gênero na gravidade dos STUI e seu impacto sobre a QV, comparamos homens e mulheres com disfunção miccional em termos de sintomas miccionais (ICSV), sintomas de incontinência (ICSI) e sintomas de freqüência/noctúria (ICSF).

\subsection{Análise estatística}

As características epidemiológicas dos pacientes foram avaliadas por meio de estatística descritiva. As correlações de Spearman foram usadas para medir a força da associação linear entre as variáveis, juntamente com os testes de Wilcoxon, Mann-Whitney e de regressão linear múltipla, conforme apropriado. Para análise da influência dos STUI e UPDRS na QV, usou-se o teste de regressão logística múltipla. O teste $\mathrm{t}$ de Student foi aplicado para comparar os escores médios da UPDRS e os diagnósticos urodinâmicos. Os dados foram processados por meio do software estatístico SPSS, versão 10. A significância estatística foi definida como $p<0,05$. 
4. RESULTADOS 


\subsection{Comprometimento neurológico devido à DP}

A avaliação neurológica dos 110 pacientes demonstrou uma média de 3,0 \pm 0,8 (intervalo de 1,5 a 5,0) na escala de HY e média de 70,0 $\pm 31,1$ (intervalo de 12 a 142), na escala de UPDRS. A duração média da doença de Parkinson foi de 12,3 \pm 7,2 anos (intervalo de um a 30).

\subsection{STUI em pacientes com DP}

A média do escore ICSmSF foi de 11,9 \pm 9,3 (intervalo de 1 a 36). A queixa urinária mais comum foi a noctúria, afetando 89 (80,9\%) pacientes.

Outros sintomas de enchimento foram: urgência em 40 (36,3\%) pacientes, freqüência urinária aumentada em 39 (35,4\%) e urge-incontinência em 23 (20,9\%) (Figura 1). Cinco pacientes (4,5\%) faziam uso de fraldas. 


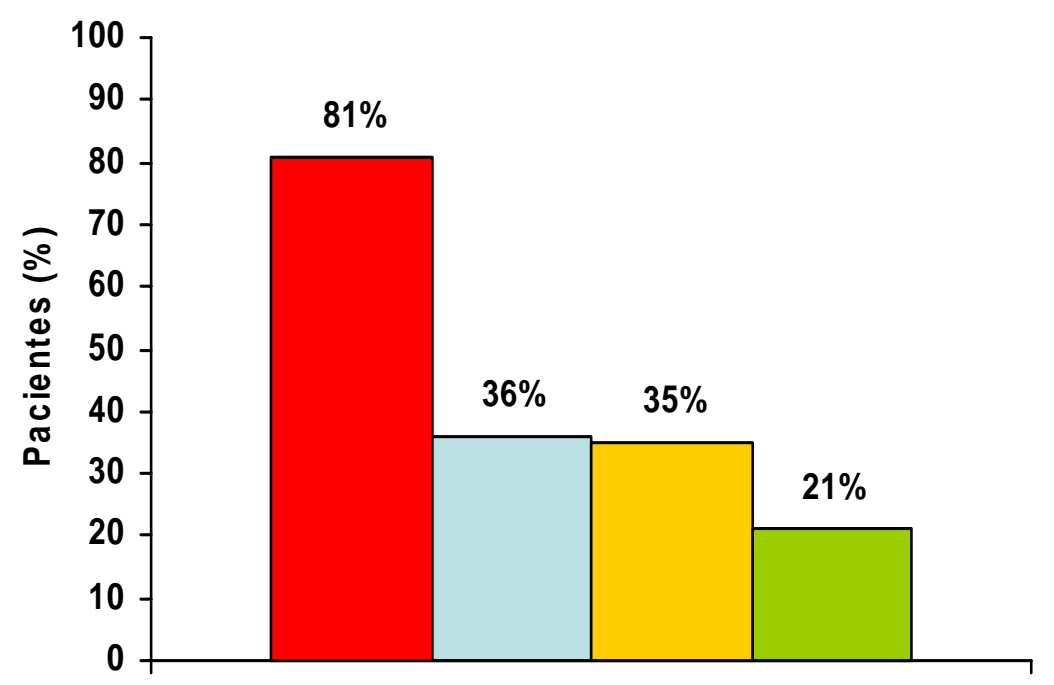

$\square$ Noctúria $\square$ Urgência $\square$ Freqüência $\square$ Urge-incontinência

Figura 1 - Prevalência dos sintomas de enchimento

Os sintomas de esvaziamento mais freqüentes foram micção intermitente em 49 (44,5\%), sensação de esvaziamento vesical incompleto em 44 (40\%), seguidos por hesitação e jato urinário fraco, ambos representados por $41(37,2 \%)$ pacientes. Esforço miccional foi o sintoma de esvaziamento menos freqüente, referido por 31 (28\%) pacientes (Figura 2). 


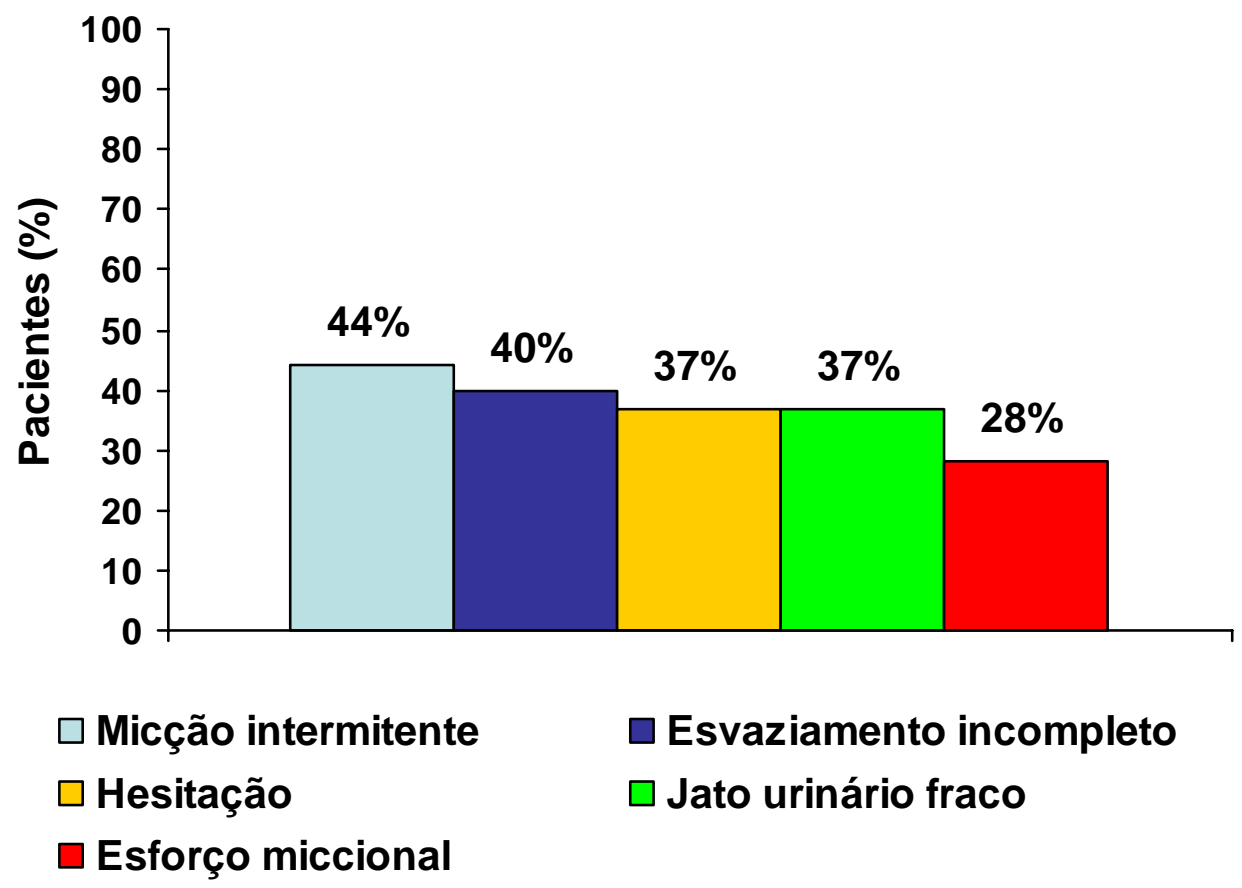

Figura 2 - Prevalência dos sintomas de esvaziamento

Os resultados do diário miccional documentam os sintomas de urgeincontinência, freqüência urinária diurna e noctúria (Figuras 3, 4 e 5).

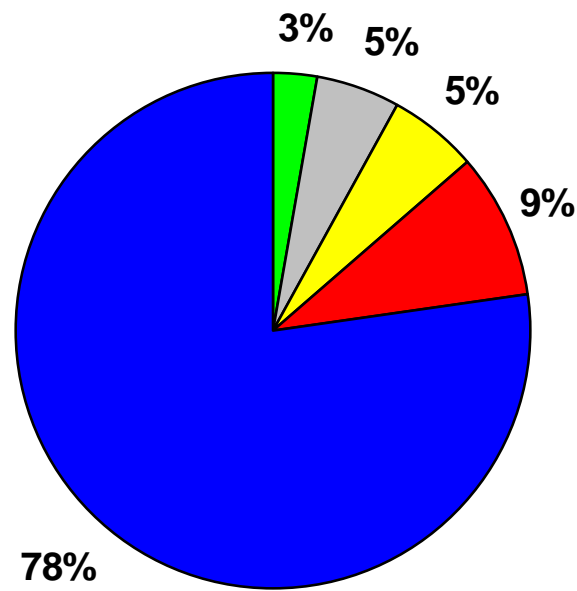

$\square 5$ ou mais $\square 3$ a $4 \quad \square 2 \square 1 \square$ Continentes

Figura 3 - Prevalência de urge-incontinência (episódios/dia) 


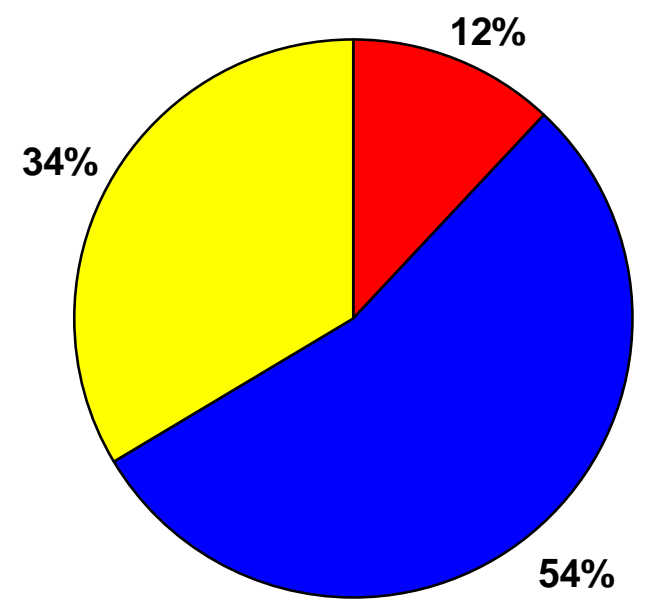

$\square 1$ a $3 \square 4$ a $7 \square 8$ ou mais

Figura 4 - Freqüência urinária diurna (micções/dia)

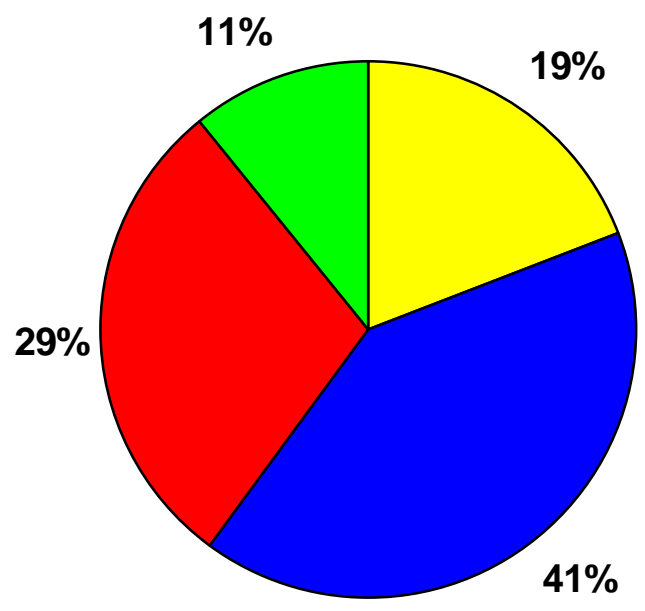

$\square 0 \square 1$ a $2 \square 3 a 4 \square 5$ ou mais

Figura 5 - Noctúria 
A duração média dos STUI variou de um a 19 anos, com média de 3,8 \pm 3,4 anos. Em 55 (50\%) pacientes, o início dos STUI ocorreu dentro de um período inferior a 7,5 anos depois do início da DP, e em 11 (10\%) pacientes, os STUI tiveram início dentro de um período inferior a um ano e meio da DP.

\subsection{Associação entre STUI e DP}

A incidência dos sintomas miccionais apresentou crescimento significante com o aumento no estágio do comprometimento neurológico. $\mathrm{Na}$ análise de regressão linear, o comprometimento neurológico medido pela escala UPDRS foi associado com disfunção miccional, medida pelo ICSmSF $(p=0,021)$ (Figura 6).

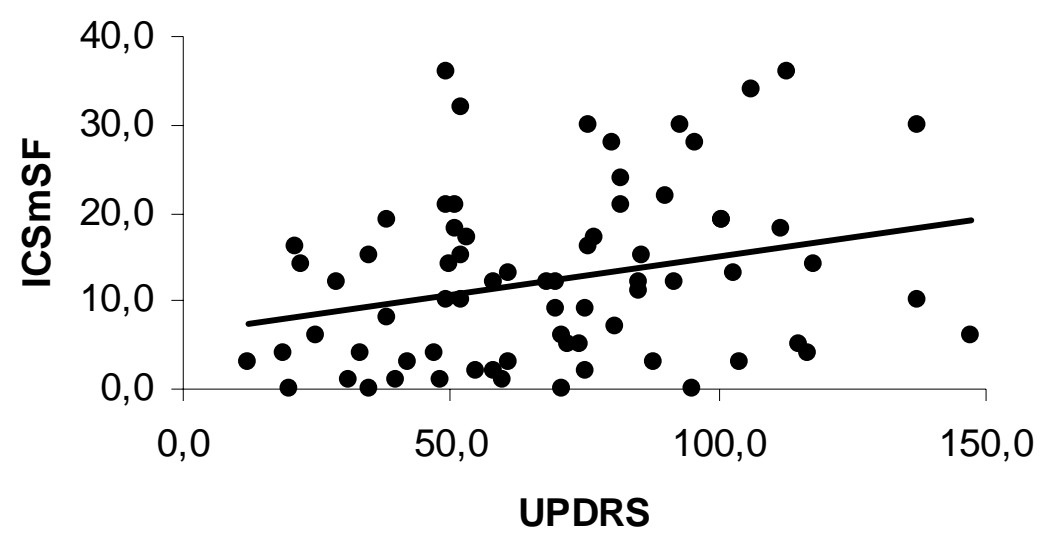

Figura 6 - Associação entre gravidade da DP (UPDRS) e sintomas do trato urinário inferior (ICSmSF) por meio de regressão linear simples $(p=0,02)$ 
A gravidade da doença medida pela escala de HY também foi associada aos STUI (rho de Spearman $=0,274, p=0,017$ ) (Figura 7). $\mathrm{Na}$ regressão linear, a gravidade dos sintomas miccionais foi significativamente associada com a duração da DP $(p=0,039)$, porém não com a idade $(p=0,809)$. Na regressão linear múltipla, no entanto, os STUI não foram significativamente influenciados pela duração da DP $(p=0,239)$.

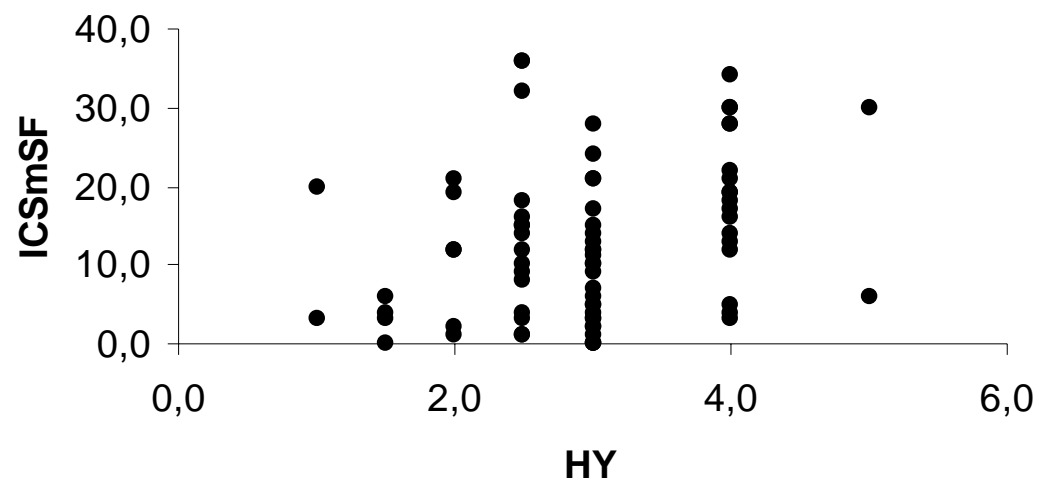

Figura 7 - Análise de correlação demonstrando efeito da gravidade da DP (HY) nos sintomas do trato urinário inferior (ICSmSF) $(p=0,01)$

\subsection{Impacto dos STUI e do comprometimento neurológico na QV (devido a sintomas urinários)}

A análise de regressão logística indicou que a QV relacionada aos sintomas urinários foi significativamente afetada pela gravidade da disfunção miccional avaliada pelo ICSmSF $(p<0,001)$. Tanto os sintomas obstrutivos 
quanto os sintomas de incontinência e de freqüência/noctúria afetaram significativamente a QV dos pacientes.

O intervalo de confiança $(95 \%)$ da razão de probabilidade indicou que os sintomas de freqüência/noctúria são melhores preditores da QV quando comparados com os de incontinência e os obstrutivos (Tabela 1). O comprometimento neurológico medido pela escala UPDRS não se correlacionou com a QV referente aos sintomas urinários (rho de Spearman $=-0,21 ; p=0,061)$.

Tabela 1 - Qualidade de vida em função dos escores de sintomas urológicos

\begin{tabular}{lccc}
\hline Sintomas & Coeficiente & Erro padrão & QV $(\boldsymbol{p})$ \\
\hline ICSV & $-0,033$ & 0,014 & 0,025 \\
ICSI & $-0,047$ & 0,019 & 0,014 \\
ICSF & $-0,301$ & 0,027 & $<0,001$ \\
\hline
\end{tabular}

\subsection{Pacientes sintomáticos}

Sessenta e três pacientes $(57,2 \%)$ foram considerados sintomáticos. Desses, 54 (11 mulheres e 43 homens) concluíram a avaliação urológica proposta e nove não completaram a avaliação por problemas de transporte ou por residirem em localidades distantes. A avaliação neurológica dos pacientes sintomáticos demonstrou um escore médio $\mathrm{HY}$ de $3,2 \pm 0,8$ (intervalo de 1,0 a 5,0) e um escore médio UPDRS de 73,8 \pm 28,7 (intervalo 
de 19 a 137). A duração média da doença foi de $12,7 \pm 7,6$ anos (intervalo de um a 30). A duração dos STUI variou de um a 19 anos, com uma média de $3,9 \pm 3,5$ anos

Comparados aos pacientes assintomáticos, os pacientes sintomáticos apresentaram maior grau de comprometimento neurológico avaliado pelo escore HY (HY de 2,8 \pm 0,3 para os assintomáticos; $p=0,035)$. Idade e duração da DP não foram significativamente diferentes entre os pacientes sintomáticos e assintomáticos.

A média ICSmSF dos pacientes sintomáticos foi de 17,1 \pm 8,6 (intervalo de três a 36). As queixas urinárias mais comuns no grupo de pacientes sintomáticos foram: noctúria em 48/54 (88,8\%), freqüência urinária aumentada em 25/54 (46,2\%), urgência em 22/54 (40,7\%) e urge-incontinência em 18/54 $(33,3 \%)$ pacientes. Três pacientes (5,5\%) faziam uso de fraldas.

Dois pacientes $(3,7 \%)$ tiveram resultado positivo na urocultura à época da avaliação e foram tratados. Os níveis de creatinina sérica e PSA estavam normais em todos os pacientes.

O volume médio da próstata, estimado por ultra-sonografia, foi de $30,3 \pm 10,7$ cc (intervalo de 18,9 a 56). O volume residual médio pósmiccional foi de $33 \pm 44 \mathrm{~mL}$ (intervalo de zero a 122). A ultra-sonografia do trato urinário foi normal em 52 pacientes e dois $(3,7 \%)$ pacientes apresentaram divertículos vesicais.

Os estudos urodinâmicos foram realizados em 54 pacientes, 43 $(79,6 \%)$ homens e $11(19,4 \%)$ mulheres. Nove pacientes sintomáticos recusaram-se a fazer os exames. A tabela 2 exibe os achados 
urodinâmicos. A hiperatividade detrusora foi detectada em 27 (50\%) pacientes. Duas pacientes do sexo feminino (18\%) apresentavam incontinência urinária de esforço.

Tabela 2 - Parâmetros urodinâmicos da doença de Parkinson

\begin{tabular}{lc}
\hline \multicolumn{1}{c}{ Parâmetro } & Média \pm DP \\
\hline Fluxo máximo $(\mathrm{mL} / \mathrm{s})$ & $11,1 \pm 6,4$ \\
Resíduo pós-miccional $(\mathrm{mL})$ & $33,1 \pm 43,8$ \\
Capacidade cistométrica $(\mathrm{mL})$ & $393,8 \pm 150,7$ \\
Complacência vesical $\left(\mathrm{mL} / \mathrm{cmH}_{2} \mathrm{O}\right)$ & $42,3 \pm 38,4$ \\
Hiperatividade detrusora* & $27(50 \%)$ \\
Fluxo máximo (mL/s) ** & $9,4 \pm 5,5$ \\
PdetQmax (cmH $\left.{ }_{2} \mathrm{O}\right)$ & $54,1 \pm 27,7$ \\
Resíduo pós-miccional $(\mathrm{mL})^{\star \star}$ & $148,6 \pm 135,8$ \\
\hline
\end{tabular}

*Número e percentual; **Estudo pressão / fluxo

Em termos gerais, a obstrução infravesical foi diagnosticada em 38 (70,3\%) pacientes, a hiperatividade detrusora em 27 (50\%) e a hipocontratilidade detrusora em seis $(11,1 \%)$ pacientes (Tabela 3). A qualidade da eletromiografia foi considerada boa em apenas 18 (33\%) pacientes e optou-se por não relatar seus resultados já que a maioria dos pacientes teve registro eletromiográfico de má qualidade. 
Tabela 3 - Achados urodinâmicos na doença de Parkinson

\begin{tabular}{ll}
\hline Achados Urodinâmicos & Número de Pacientes (\%) \\
\hline Normal & $3(5,6)$ \\
Hipocontratilidade detrusora & $4(7,4)$ \\
HD e Hipocontratilidade detrusora & $2(3,7)$ \\
Hiperatividade detrusora & $7(12,9)$ \\
HD e OIV & $18(33,4)$ \\
Obstrução infravesical & $20(37,0)$ \\
\hline Total $n^{\circ}(\%)$ & $54(100)$ \\
\hline
\end{tabular}

\subsection{Associação entre anormalidades urodinâmicas e comprometimento neurológico}

O comprometimento neurológico medido pela escala UPDRS não foi capaz de prever os achados de hiperatividade detrusora e/ou obstrução infravesical, indicando que as anormalidades urodinâmicas na DP podem ser independentes da gravidade da doença (Tabela 4).

Tabela 4 - Associação entre achados urodinâmicos e comprometimento neurológico

\begin{tabular}{lccc}
\hline & Presente* $^{*}$ & Ausente* $^{*}$ & $\boldsymbol{p}$ \\
\hline Hiperatividade detrusora & $71,9 \pm 29,7$ & $79,7 \pm 22,8$ & 0,551 \\
Obstrução infravesical & $78,8 \pm 28,8$ & $64,1 \pm 26,7$ & 0,120 \\
HD e OIV & $67,8 \pm 30,3$ & $83,8 \pm 22,1$ & 0,099 \\
\hline
\end{tabular}

* Escala UPDRS (Média \pm Desvio Padrão) 


\subsection{STUI e QV em homens e mulheres com DP}

Não se observaram diferenças significativas na comparação entre pacientes do sexo masculino e feminino em termos de idade, comprometimento neurológico, duração da DP e duração dos STUI. Além disso, a gravidade dos sintomas miccionais, assim como seu impacto na QV, não foi significativamente diferente entre homens e mulheres. A diferença observada foi um maior índice de sintomas miccionais (ICSV) em pacientes do sexo masculino (Tabela 5). Foi interessante notar que a prevalência de obstrução infravesical não foi significativamente diferente entre homens e mulheres $(74,4 \%$ e $54,5 \%$, respectivamente; $p=0,270)$.

Tabela 5 - Parâmetros clínicos, STUI e qualidade de vida em homens e mulheres com doença de Parkinson

\begin{tabular}{lccc}
\hline & Homens & Mulheres & $\boldsymbol{p}$ \\
\hline Idade* & $60,4 \pm 7,5$ & $64,4 \pm 12,6$ & 0,328 \\
UPDRS* & $74,2 \pm 22,2$ & $72,6 \pm 43,8$ & 0,915 \\
Duração da DP** & $12(4,0-30,0)$ & $7,5(1,0-20,0)$ & 0,154 \\
Duração dos STUI** & $3,0(1,0-19,0)$ & $2,5(1,0-5,5)$ & 0,287 \\
ICSmSF* & $17,4 \pm 7,9$ & $16,2 \pm 10,7$ & 0,678 \\
ICSV* & $7,0 \pm 4,6$ & $3,8 \pm 4,0$ & 0,045 \\
ICSI** & $5,0(0-18,0)$ & $5,0(0-20,0)$ & 0,530 \\
ICSF* & $5,6 \pm 2,7$ & $5,5 \pm 2,6$ & 0,922 \\
QV** & $2,0(0-3,0)$ & $2,0(1,0-3,0)$ & 0,531 \\
\hline
\end{tabular}

*Escala (média \pm desvio padrão); **Mediana (valor mínimo - máximo) 
5. DISCUSSÃO 
Distúrbios miccionais são freqüentes em pacientes com DP, com prevalência estimada em 27\% a 78\% (Araki e Kuno, 2000; Sakakibara et al., 2001b; Campos-Sousa et al., 2003). Vários fatores contribuem para a elevada prevalência de STUI nos pacientes com DP incluindo alterações vesicoesfincterianas secundárias à doença neurológica, comorbidades do aparelho urinário e alterações degenerativas do trato urinário associadas ao envelhecimento. Assim, torna-se difícil estabelecer o papel desempenhado para cada um desses fatores na gênese dos STUI.

Neste trabalho prospectivo, estudamos de forma abrangente uma população de pacientes com DP, com o objetivo inicial de investigar a prevalência e as características dos STUI. Foram avaliados 110 pacientes, que foram convidados a participar do estudo independentemente da presença de STUI, incluindo $84(76,3 \%)$ homens e $26(23,7 \%)$ mulheres. A maioria dos pacientes avaliados apresentava doença neurológica em grau 
moderado e observou-se que a prevalência da disfunção miccional aumentou significativamente quanto maior a gravidade da DP.

Nossos resultados demonstraram que os STUI, principalmente o aumento da freqüência urinária e noctúria, têm impacto negativo na QV dos pacientes. Ao contrário de outros estudos em que a hiperatividade detrusora é a anormalidade urodinâmica mais comum, a obstrução infravesical foi o achado mais freqüente e o grau de comprometimento neurológico não apresentou associação com os achados urodinâmicos. Finalmente, praticamente não encontramos diferenças entre os STUI e anormalidades urodinâmicas afetando homens e mulheres.

A maior parte dos estudos envolvendo pacientes com STUI e DP demonstra predomínio dos pacientes do sexo masculino (Pavlakis et al., 1983; Stocchi et al., 1997; Winge et al., 2005). Na DP, a proporção entre homens e mulheres na maioria dos estudos epidemiológicos é estimada em 2:1 (Baldereschi et al., 2000; Van Den Eeden et al., 2003; Baba et al., 2005), inferior à que encontramos. No presente estudo, observamos a proporção aproximada de 3:1 que corresponde à real proporção de homens e mulheres atendidos no ambulatório de Neurologia do HC-FMUSP (Dias, 2006). Isso indica um possível viés de procura por assistência médica em nossa instituição.

Entre os fatores possivelmente relacionados aos distúrbios miccionais, avaliamos a idade, o tempo de duração da DP e o grau de comprometimento neurológico da doença. Optamos por não avaliar os possíveis efeitos do uso de drogas anti-parkinsonianas nos STUI e nos 
parâmetros urodinâmicos, já que todos os pacientes estudados faziam uso de várias medicações pertencentes a diferentes classes de drogas antiparkinsonianas, como drogas dopaminérgicas (levodopa, carbidopa, bromocriptina), anticolinérgicas (biperideno), inibidores da mono-aminooxidase tipo B (selegiline), inibidores da catechol-O-metiltransferase (tolcapone e entacapone) e outras. Além disso, muitos pacientes com DP recebem dosagens diferentes dos medicamentos e outras medicações para alterações cognitivas e psiquiátricas que freqüentemente os acompanham.

A gravidade da DP foi avaliada tanto pela escala de HY como pela escala UPDRS. Utilizamos as duas escalas em razão das diferenças existentes entre as mesmas. A escala de HY é mais simples e de rápida aplicação e vem sendo utilizada para classificar o grau de comprometimento neurológico de pacientes com DP há vários anos. A escala UPDRS foi desenvolvida recentemente e é mais completa e abrangente. Embora exista boa correlação entre as duas escalas, a escala UPDRS é considerada mais acurada para avaliar os distúrbios neurológicos da DP, mas sua aplicação é mais trabalhosa, pois envolve vários itens. Ambas têm sido utilizadas nos estudos que avaliam os distúrbios miccionais em pacientes com DP (Aranda, 1993; Dmochowski, 1999; Araki et al., 2000; Campos-Sousa et al., 2003).

No presente estudo, o escore médio de HY foi três, variando de um a cinco. A maior parte dos pacientes avaliados apresentava a forma moderada da doença. As formas leves ou muito graves foram raras nesta amostra, incluindo somente dois pacientes com HY igual a cinco, que representa a forma mais avançada da DP. 
Os sintomas miccionais foram avaliados pelo questionário ICSmSF. Trata-se de um questionário de sintomas estruturado e de fácil compreensão, contendo questões pertinentes aos sintomas miccionais (ou de esvaziamento), de incontinência, freqüência e noctúria, além de uma questão específica que avalia a qualidade de vida em relação aos sintomas miccionais. Optamos por este questionário por considerá-lo mais abrangente que o International Prostate Score Symptoms (IPSS) e por ser recomendado pela International Continence Society (Sociedade Internacional de Continência) (Abrams et al., 2002). Trata-se, portanto, de um questionário mais abrangente e detalhado do que o IPSS (Barry et al., 1992), que é o mais utilizado na maioria dos estudos.

Os sintomas miccionais mais freqüentes encontrados neste estudo foram noctúria, aumento da freqüência miccional e urgência miccional, o que está de acordo com outros estudos que avaliaram STUI na DP (Andersen e Bradley, 1976; Pavlakis et al., 1983; Hattori et al., 1992; Araki e Kuno, 2000; Lemack et al., 2000; Sakakibara et al., 2001b; Campos-Sousa et al., 2003).

Metade dos pacientes avaliados apresentou disfunção miccional dentro dos primeiros 7,5 anos após o diagnóstico da DP. Um pequeno percentual, correspondendo a $10 \%$ da amostra, apresentou STUI um ano e meio após o início da DP. Habitualmente, o estabelecimento das disfunções miccionais na DP ocorre nos primeiros seis anos após o início dos sintomas motores (Andersen e Bradley, 1976; Shimizu et al., 1997).

A prevalência dos sintomas miccionais aumentou significativamente quanto maior a gravidade da DP nos pacientes, que foram avaliados tanto 
pelo escore HY como pelo UPDRS. Também observamos a associação dos sintomas miccionais com o tempo de duração da doença que, entretanto, não foi confirmada na regressão linear múltipla. Esses achados confirmam os resultados de outros estudos que demonstraram o aumento dos STUI conforme a progressão da DP (Hattori et al., 1992; Araki e Kuno, 2000), mas contraria um estudo que demonstrou associação entre STUI e idade dos pacientes (Campos-Sousa et al., 2003). A exemplo do presente trabalho, a maioria dos autores não demonstrou associação entre tempo de duração da DP e prevalência dos STUI (Araki e Kuno, 2000; Campos-Sousa et al., 2003; Hobson et al., 2003; Winge et al., 2006).

A DP é mais freqüente em pacientes com idade superior a 60 anos (Baldereschi et al., 2000; Louis et al., 2004), que representa uma faixa etária mais sujeita a sofrer distúrbios miccionais. Não se sabe em quais pacientes com DP os sintomas miccionais são atribuídos predominantemente às alterações vesicoesfincterianas secundárias à doença neurológica, e em quais pacientes outros fatores são mais importantes, como comorbidades do aparelho urinário e alterações degenerativas do trato urinário associadas ao envelhecimento. Neste estudo, não houve associação significativa entre disfunção miccional e idade dos pacientes. Resultados semelhantes foram relatados por Hobson et al. (2003), que avaliaram 123 pacientes com DP e também não observaram essa associação. Sabe-se que, na população geral, existe associação positiva entre idade e presença de sintomas miccionais. O fato de não encontrarmos associação em pacientes com DP provavelmente reflete o impacto de dois fatores: primeiro, a faixa etária dos 
pacientes ser relativamente restrita, pelo fato de a doença acometer principalmente indivíduos com mais de 55-60 anos; segundo, pelo fato de que a doença neurológica tem repercussões muito significativas sobre o controle miccional de muitos pacientes, sobrepondo-se ao efeito da idade.

O efeito negativo dos STUI na QV dos pacientes foi avaliado por uma única questão em que o paciente responde "quanto seus sintomas urinários interferem na sua vida?" (Donovan et al., 2000). As repostas possíveis são nem um pouco, um pouco, moderadamente e bastante, sendo pontuadas de zero a três, respectivamente. Observamos correlação entre os sintomas miccionais e a qualidade de vida específica (relacionada à função miccional), com os pacientes mais sintomáticos apresentando pior QV. Araki et al. (2000) também observaram associação entre os sintomas miccionais e a QV em pacientes com DP. Magerkurth et al. (2005) avaliaram o efeito de variados sintomas autonômicos (vesicais, intestinais, cardiovasculares e sudorese) sobre as atividades de vida diária de pacientes parkinsonianos. Cerca de $50 \%$ dos pacientes avaliados classificaram como moderado a severo o impacto dos sintomas autonômicos nas suas atividades cotidianas.

Procuramos caracterizar quais sintomas miccionais apresentavam maior impacto na qualidade de vida dos pacientes. Observamos que os sintomas de aumento da freqüência e noctúria exerceram maior impacto negativo na QV dos pacientes em comparação com os sintomas miccionais (de esvaziamento) e os sintomas de incontinência. Resultados muito semelhantes foram relatados por Araki et al. (2000), que observaram 
associação entre os escores de QV e IPSS, demonstrando que os sintomas irritativos (de enchimento) são os que mais incomodam os pacientes.

Observamos uma tendência à associação entre o grau de comprometimento neurológico e a qualidade de vida em relação ao STUI, o que provavelmente reflete a associação entre a gravidade da DP e os sintomas miccionais.

$\mathrm{Na}$ segunda fase do presente estudo, avaliamos os pacientes considerados sintomáticos em relação à função miccional. Foram considerados sintomáticos aqueles desejosos de tratamento, independentemente da quantificação dos sintomas miccionais pelo questionário ICSmSF. Sessenta e três pacientes foram definidos como sintomáticos, dos quais 54 completaram a avaliação proposta. Os pacientes sintomáticos e os assintomáticos não tiveram diferenças significantes em termos de idade e tempo de duração da DP. Houve maior comprometimento neurológico nos pacientes sintomáticos. Esses achados reforçam a importância do grau de comprometimento neurológico sobre os sintomas miccionais e o menor impacto de fatores como idade e tempo de duração da doença sobre os mesmos.

A influência de fatores como idade, grau de comprometimento neurológico e tempo de duração da DP sobre o trato urinário é muito controversa. Possivelmente, isso ocorre pela interdependência desses parâmetros e por ser a DP uma doença progressiva, existindo uma correlação entre severidade da doença neurológica e o tempo de duração da DP. Da mesma forma, pacientes com idade mais avançada tendem a apresentar DP com maior tempo de duração. Nossos achados reforçam a 
importância da gravidade da DP como único fator preditivo isolado para o desenvolvimento da disfunção miccional.

A avaliação do volume prostático em homens com DP e disfunção miccional pode ser importante já que a prevalência de hiperplasia benigna e outras doenças da próstata é elevada em pacientes dessa faixa etária. Apesar disso, não encontramos na literatura médica dados de volume prostático nos estudos que avaliaram os distúrbios miccionais em pacientes parkinsonianos. Na nossa série, o volume médio da próstata estimado por ultra-sonografia foi de 30,3 cc, que é compatível com ou até menor que a média de volume prostático da população geral na mesma faixa etária (Roehrborn et al., 1999; Hassler et al., 2001). Esse achado indica que, em nossa amostra, a hiperplasia da próstata não foi um fator importante a contribuir para os sintomas miccionais.

Nos pacientes sintomáticos, os achados urodinâmicos mais freqüentes foram obstrução infravesical, hiperatividade detrusora e hipocontratilidade detrusora, acometendo respectivamente 70,4\%, 50\% e 11\% dos pacientes estudados. Apenas três pacientes apresentaram estudo urodinâmico normal.

Nos trabalhos que avaliaram as anormalidades urodinâmicas na DP, diferentes métodos e definições foram utilizados, o que prejudica uma tentativa de comparação desses estudos. O método de enchimento vesical, que pode ter impacto nos resultados da cistometria, variou entre os autores, sendo que alguns realizaram enchimento com $\mathrm{CO}_{2}$ (Andersen e Braldey, 1976; Pavlakis, 1983) enquanto outros utilizaram soro fisiológico (Stocchi et al., 
1997; Araki et al., 2000). A definição urodinâmica de hiperatividade detrusora também foi diferente entre os autores. Alguns consideraram qualquer contração detrusora involuntária durante o enchimento como HD (Aranda, 1993; Araki et al., 2000). Contudo, outros trabalhos consideraram somente as contrações detrusoras com Pdet > 15 cmH2O (Stocchi et al., 1997; Bonnet et al., 1997). Na avaliação da fase miccional, as diferenças de metodologia são ainda mais acentuadas. A atividade esfincteriana foi avaliada através de eletromiografia com agulha (Pavlakis et al., 1983; Stocchi et al.,1997), eletromiografia com eletrodos de superfície (Seif et al., 2003), cateter de membrana (Bonnet et al., 1997), vídeo-urodinâmica (Dmochowski, 1999; Sakakibara et al., 2001a) ou estudo pressão-fluxo convencional (Araki et al., 2000; Sakakibara et al., 2001a). Além disso, a definição de OIV variou entre os autores que avaliaram a fase miccional com o mesmo método.

No presente estudo as definições e a metodologia do exame urodinâmico utilizadas seguiram as normas da ICS (Abrams et al., 2002). Assim, consideramos como hiperatividade detrusora a presença de contrações detrusoras involuntárias, de qualquer magnitude, durante a fase de enchimento. Avaliamos a fase de esvaziamento vesical dos pacientes com eletromiografia de superfície e estudo pressão/fluxo. Pelas limitações técnicas deste tipo de eletromiografia e por termos tido mais de metade dos exames com má qualidade de registro eletromiográfico, preferimos dar ênfase aos resultados dos estudos pressão/fluxo. Optamos por classificar os pacientes segundo o nomograma de Schäfer (Schäfer et al., 1989; Schäfer, 1990), pela sua ampla aceitação e por também avaliar a contratilidade 
detrusora. Observamos $70,3 \%$ de pacientes com obstrução infravesical. A maioria dos estudos que utilizaram o estudo urodinâmico para avaliar o esvaziamento vesical de pacientes parkinsonianos discute principalmente os achados da função esfincteriana, geralmente avaliada por eletromiografia de agulha e/ou vídeo-urodinâmica, tendo relatado obstrução em 27\% a 58\% dos pacientes por dissinergismo vesicoesfincteriano ou bradicinesia esfincteriana (De Marinis et al., 1993; Stocchi et al., 1997; Araki et al., 2000). Poucos trabalhos avaliaram o estudo miccional através de dados de pressão detrusora, fluxo e nomogramas de obstrução. Esse fato é compreensível, já que se acredita que a obstrução infravesical na maioria dos pacientes com DP seja secundária a problemas esfincterianos como dissinergismo e bradicinesia. Além disso, os nomogramas foram desenvolvidos para avaliação de pacientes com hiperplasia prostática benigna, e sua aplicação em pacientes parkinsonianos pode não ser apropriada. Entretanto, os estudos pressão/fluxo são mais universalmente utilizados e não requerem equipamentos e conhecimentos especializados como os necessários para a realização de eletromiografia de agulha. Além disso, fornecem índices objetivos de obstrução vesical e contratilidade detrusora que são úteis para a avaliação de pacientes com disfunções miccionais neurogênicas (Sakakibara et al., 2000; Sakakibara et al., 2001a).

Nossos resultados do estudo miccional não apontaram diferença significativa na proporção de homens e mulheres obstruídos. É possível que, caso tivéssemos avaliado maior contingente de pacientes do sexo feminino, a diferença observada pudesse ter sido significativa. De qualquer 
forma, o achado de obstrução em 74,4\% dos homens e 54,5\% das mulheres é bastante elevado e indica que o padrão miccional de pacientes com DP e disfunção miccional é obstrutivo na maioria dos casos, de acordo com nomograma de obstrução desenvolvido para homens com HPB. Sakakibara et al. (2001a) usaram o número de Abrams-Griffiths para avaliar 15 homens e seis mulheres com DP. O número de Abrams-Griffiths é derivado do nomograma de Abrams-Griffiths e considera obstruídos os homens com valores maiores que 40 (Abrams, 1999). No referido estudo, a média do número de Abrams-Griffiths para pacientes com DP foi 40 em mulheres e 43 em homens. Assim, os achados de Sakakibara et al. (2001a) estão de acordo com os de nossa série, por demonstrarem que grande parte dos pacientes está obstruída, e que homens e mulheres apresentam padrão miccional semelhante. Como a fisiopatologia da obstrução urinária nesses pacientes está geralmente relacionada ao mau funcionamento do esfíncter estriado, o achado de obstrução infravesical semelhante em homens e mulheres não surpreende.

A prevalência de hiperatividade detrusora em nosso estudo foi semelhante à encontrada por outros autores, tendo sido reportada em $46 \%$ a 88\% dos pacientes avaliados (Aranda, 1993; Araki et al., 2000; Sakakibara et al., 2001a; Sakakibara et al., 2001b). Corresponde à anormalidade urodinâmica mais freqüente em parkinsonianos e pode explicar a elevada prevalência de sintomas de enchimento nesses pacientes, como aumento da freqüência, urgência e noctúria. Acredita-se que os gânglios da base, incluindo a substância nigra, tenham efeito inibitório sobre o reflexo da 
micção (Lewin et al., 1967). Dessa forma, a degeneração de seus neurônios dopaminérgicos poderia explicar a elevada prevalência de hiperatividade detrusora em parkinsonianos (Alvord et al., 1974). Por outro lado, o fato de que a hiperatividade detrusora não está associada à severidade da doença de Parkinson (achado deste e de outros estudos) (Pavlakis et al.,1983; Stocchi et al., 1997; Araki et al., 2000) enfraquece um pouco essa hipótese.

Hipocontratilidade detrusora foi encontrada em 11\% dos pacientes avaliados, taxa semelhante à de outros estudos, nos quais variou de três a 16\% (Pavlakis et al.,1983; Araki et al., 2000). Araki et al. (2000) observaram que o padrão de hipocontratilidade associada à hiperatividade detrusora foi mais comum em pacientes com doença neurológica mais grave. Esse padrão urodinâmico pode ser encontrado em contingente significativo de pacientes com idade avançada, e parece ser secundário a alterações degenerativas severas da bexiga (Resnick e Yalla, 1987).

Procuramos correlacionar os achados urodinâmicos de obstrução infravesical e hiperatividade detrusora com o grau de comprometimento neurológico da DP. A falta de correlação entre esses parâmetros indica que as anormalidades urodinâmicas nos pacientes parkinsonianos são independentes da severidade da doença neurológica. Assim, o exame urodinâmico é essencial quando se quer identificar precisamente a natureza da disfunção miccional nesses pacientes. Resultados semelhantes foram encontrados por Araki et al. (2000), que somente identificaram maior prevalência da associação entre hipocontratilidade detrusora e hiperatividade detrusora e as formas mais avançadas da DP. 
Dado interessante deste estudo foi a semelhança encontrada entre homens e mulheres em praticamente todos os parâmetros estudados. Ambos os sexos apresentaram idade, duração da DP, gravidade da DP e duração dos STUI muito semelhantes. Além disso, a gravidade dos STUI e o impacto na QV foram iguais nos dois grupos. A diferença observada foi a maior intensidade dos sintomas de esvaziamento nos homens. Entretanto, não observamos diferença significativa na prevalência de obstrução infravesical no exame urodinâmico dos homens e mulheres. Araki e Kuno (2000) encontraram resultados semelhantes em estudo epidemiológico, através da avaliação dos pacientes com o IPSS. Demonstraram que os sintomas de esvaziamento vesical foram mais intensos nos homens, enquanto o escore total e os sintomas irritativos não apresentaram diferenças em comparação com as mulheres.

Concluindo, a avaliação urológica revelou uma elevada prevalência de sintomas do trato urinário inferior numa expressiva amostra de pacientes com DP. Os sintomas urinários associam-se à gravidade da DP, mas não ao sexo e à idade dos pacientes. A qualidade de vida é prejudicada pelos sintomas miccionais. Nos pacientes significativamente sintomáticos, a obstrução infravesical e a hiperatividade detrusora são os achados urodinâmicos mais comuns e não estão correlacionados com a gravidade da doença neurológica. 


\section{CONCLUSÕES}


1. Sintomas do trato urinário inferior são freqüentes na doença de Parkinson, podendo surgir em sua apresentação inicial. Noctúria foi o sintoma mais comum.

2. Os sintomas do trato urinário inferior apresentaram impacto negativo na qualidade de vida dos pacientes. A gravidade da doença neurológica é o único fator preditivo isolado para a ocorrência de disfunção miccional.

3. Obstrução infravesical e hiperatividade detrusora foram os achados urodinâmicos mais freqüentes. Os achados urodinâmicos não se associam com a gravidade da doença neurológica.

4. A única diferença observada entre homens e mulheres foi a maior prevalência de sintomas de esvaziamento nos pacientes do sexo masculino. Não houve diferenças quanto ao impacto na qualidade de vida e aos achados urodinâmicos. 
7. ANEXOS 


\section{ANEXO A}

\section{ESCALA DE HOEHN E YAHR}

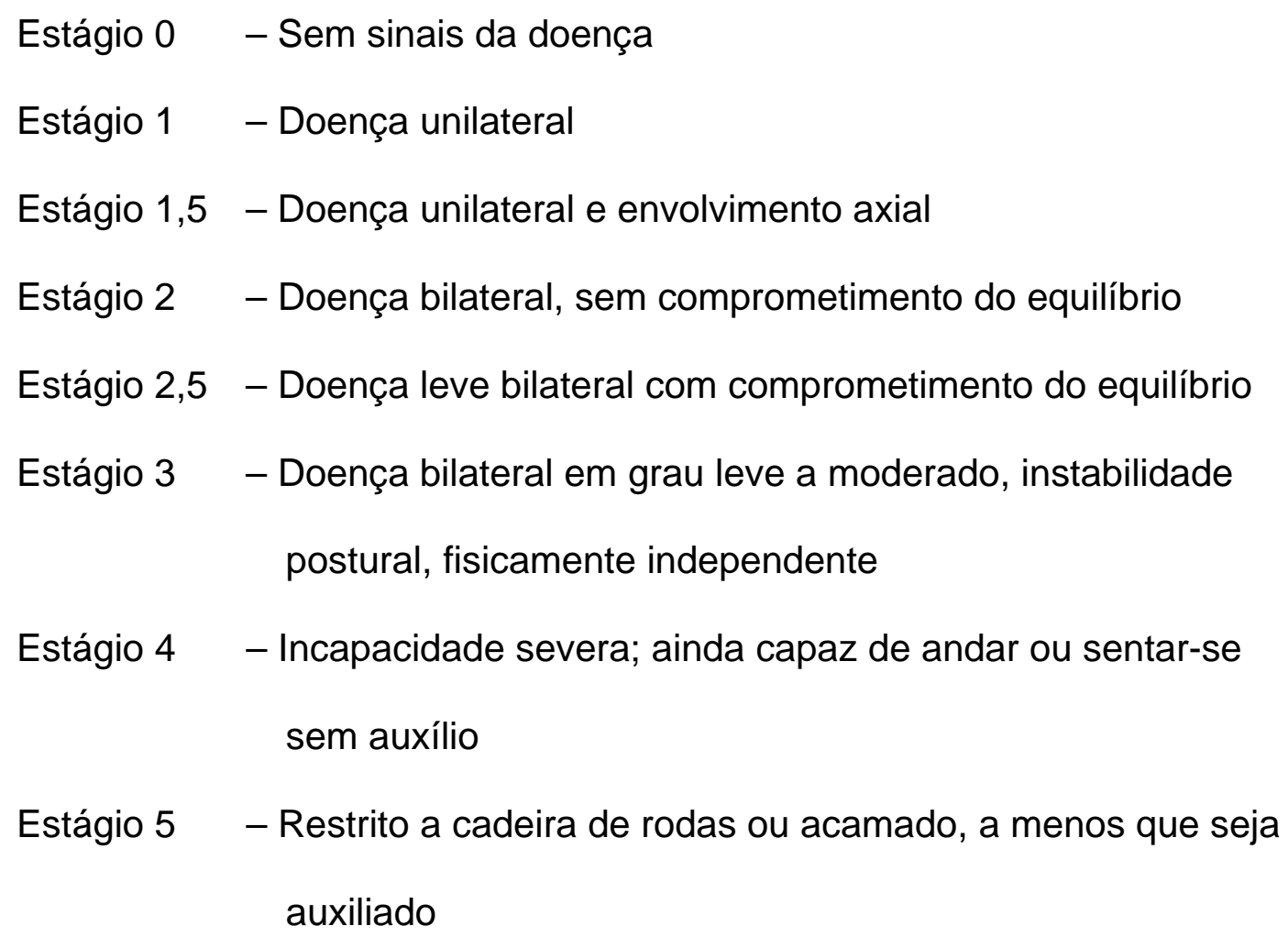




\section{ANEXO B}

\section{ESCALA DE CLASSIFICAÇÃO UNIFICADA DA DOENÇA DE PARKINSON}

\section{I - RACIOCÍNIO, COMPORTAMENTO E HUMOR}

\section{Comprometimento intelectual}

$0=$ Nenhum.

$1=$ Leve. Esquecimento persistente com recordação parcial dos eventos e sem outras dificuldades.

2 = Moderada perda da memória, desorientação e dificuldade para resolver problemas mais complexos. Prejuízo das funções domiciliares e rotineiras, com necessidade de estímulo para executálas.

3 = Perda acentuada da memória com desorientação no tempo e espaço Acentuada dificuldade em resolver problemas.

4 = Perda severa da memória com orientação preservada somente para pessoas. Incapaz de fazer julgamentos e resolver problemas.

Precisa de auxílio e supervisão constante. Não pode ficar sozinho.

\section{Desordens do pensamento (em decorrência da demência ou} intoxicação por drogas)

$0=$ Nenhum.

$1=$ Vive sonhando.

2 = Alucinações e introspecção.

3 = Alucinações e desilusões freqüentes, sem introspecção, podendo interferir nas atividades rotineiras.

4 = Alucinações freqüentes, desilusões, psicose. Incapaz de ficar sozinho.

\section{Depressão}

1 = Períodos de tristeza ou culpa maiores que o normal, nunca superiores a dias ou semanas.

2 = Depressão prolongada (igual ou superior a uma semana).

3 = Depressão prolongada com sintomas vegetativos (insônia, anorexia, perda do peso, desinteresse).

4 = Depressão prolongada com sintomas vegetativos associados a pensamentos e intenções suicidas.

\section{Motivação e iniciativa}

$1=$ Normal.

$2=$ Menos afirmativo e mais passivo que o usual.

$3=$ Perda da iniciativa e desinteresse pelas atividades rotineiras.

4 = Fechado em si mesmo, completa perda da motivação. 


\section{II - ATIVIDADES COTIDIANAS}

\section{Fala}

$0=$ Normal.

$1=$ Pouco afetada. Sem dificuldades para ser compreendido.

2 = Moderadamente afetada. Às vezes a pronúncia não é compreendida.

$3=$ Severamente afetado. Freqüentemente é solicitado a repetir o que falou.

4 = Não é compreendido a maior parte do tempo.

\section{Salivação}

$0=$ Normal.

1 = Discreto aumento da salivação com episódios de perdas durante a noite.

2 = Salivação moderada com mínima perda.

$3=$ Salivação excessiva com perda discreta.

4 = Não contém a salivação e necessita de lenços constantemente.

\section{Deglutição}

$0=$ Normal.

$1=$ Raramente engasga.

2 = Engasga ocasionalmente.

$3=$ Requer alimentos macios.

4 = Requer sonda nasogástrica ou alimentação por gastrostomia.

\section{Escrever a mão}

$0=$ Normal.

$1=$ Escreve lentamente com letras pequenas.

$2=$ Moderadamente lento ou com letras pequenas; todas as palavras são legíveis.

3 = Afetado severamente; nem todas as palavras são legíveis.

4 = A maioria das palavras não é legível.

\section{Cortar alimentos e manusear utensílios domésticos}

$0=$ Normal.

$1=$ Lento e com dificuldades, mas não necessita de ajuda.

2 = Pode cortar a maioria dos alimentos, porém lentamente e com dificuldade. Precisa de algum auxílio.

$3=$ Os alimentos devem ser cortados por alguém. Consegue comer lentamente.

$4=$ Necessidade de ser alimentado. 


\section{Vestir-se}

$0=$ Normal.

$1=$ Lento, sem precisar de auxílio.

2 = Ocasional assistência para abotoar ou colocar os braços nas mangas.

$3=$ Precisa de considerável ajuda, mas pode fazer alguma coisa sozinho.

$4=$ Precisa de auxílio constantemente.

\section{Higiene}

$0=$ Normal.

$1=$ Lento, sem precisar de auxílio.

2 = Precisa de auxílio para banhar-se ou usar o sanitário, ou muita lentidão nos cuidados de higiene.

$3=$ Requer assistência para lavar-se, escovar os dentes, pentear cabelos e ir ao toalete.

4 = Cateter de Foley ou outras formas de auxílio mecânico.

\section{Virar-se na cama e ajustar as roupas de cama}

$0=$ Normal.

$1=$ Lento e com dificuldades, sem precisar de ajuda.

2 = Pode virar-se sozinho e ajustar os lençóis, mas com muita dificuldade.

3 = Pode iniciar, mas não consegue virar ou ajustar lençóis sem auxílio.

$4=$ Precisa de auxílio constantemente.

\section{Quedas}

$0=$ Nenhuma.

$1=$ Cai raramente.

2 = Cai ocasionalmente, menos que uma vez por dia.

3 = Cai em média uma vez por dia.

4 = Cai mais que uma vez por dia.

\section{Sensação de frio ao andar}

$0=$ Nenhuma.

1 = Raramente sente frio ao andar.

$2=$ Frio ocasional ao andar.

3 = Freqüentemente com frio. Ocasionalmente cai em decorrência da sensação de congelamento.

4 = Freqüentemente cai em decorrência do frio.

\section{Marcha}

$0=$ Normal.

1 = Lento e com dificuldades. Pode não balançar os braços e tender a arrastar as pernas.

$2=$ Moderada dificuldade, mas requer pouca ou nenhuma assistência.

3 = Distúrbio grave da marcha, precisando de assistência.

4 = Não pode andar, somente com assistência. 
16. Tremor (Queixa-se de tremores em alguma parte do corpo)

$0=$ Ausente.

$1=$ Desprezível e poucas vezes presente.

$2=$ Moderado e que incomoda o paciente.

$3=$ Severo e interfere em muitas atividades.

$4=$ Intenso e interfere na maior parte das atividades.

\section{Queixas sensoriais relacionadas ao parkinsonismo}

$0=$ Ausente.

$1=$ Ocasionalmente apresenta fraqueza, formigamentos ou dores leves.

2 = Freqüentemente apresenta fraqueza, formigamentos ou dores leves que não são agonizantes.

$3=$ Sensações de tormento e dores freqüentes.

$4=$ Dor excruciante.

\section{III - EXAME DOS MOVIMENTOS}

\section{Fala}

$0=$ Normal.

1 = Discreta perda da expressão, dicção e/ou volume.

2 = Monotonia, falar gaguejando, moderadamente prejudicado.

$3=$ Marcadamente comprometido, difícil de compreender.

$4=$ Incompreensível.

19. Expressão facial

$0=$ Normal.

1 = Mínima hiponímia, pode ser normal.

$2=$ Discreta, mas definitivamente com diminuição da expressão facial.

$3=$ Moderada hiponímia, às vezes com lábios partidos.

4 = Fácies fixa com severa ou completa perda da expressão facial, lábios partidos.

\section{Tremor de repouso}

$0=$ Ausente.

$1=$ Discreto e presente raramente.

2 = Leve em amplitude e persistente, ou pode ser moderado em amplitude, mas presente somente na forma intermitente.

$3=$ Moderado em amplitude e presente na maioria das vezes.

$4=$ Acentuado em amplitude e presente na maior parte do tempo.

\section{Ação ou tremor postural das mãos}

$0=$ Ausente.

$1=$ Discreto, presente com ação.

$2=$ Moderado em amplitude, presente com ação.

3 = Moderado em amplitude com postura de segurar, enquanto em ação.

4 = Acentuado em amplitude, interfere na alimentação. 
22. Rigidez (considerado no movimento passivo das maiores articulações com paciente relaxado e sentado)

$0=$ Ausente.

$1=$ Discreto ou detectável somente quando ativado por reflexos ou outros movimentos.

$2=$ Leve para moderado.

3 = Acentuado, mas grande parte dos movimentos é executável.

4 = Severo; os movimentos são realizados com dificuldade.

23. Movimentos com os dedos (paciente faz movimentos/tapas com o dedo indicador numa rápida sucessão)

$0=$ Normal.

$1=$ Um pouco lento e com redução na amplitude.

$2=$ Moderadamente comprometido. Cansa precocemente. Podem ocorrer movimentos arrastados ocasionais.

$3=$ Prejudicado severamente. Hesitação freqüente ao iniciar os movimentos.

$4=$ Dificilmente realiza os movimentos.

24. Movimento das mãos (Paciente abre e fecha as mãos numa rápida sucessão)

$0=$ Normal.

$1=$ Um pouco lento e com redução na amplitude.

$2=$ Moderadamente comprometido. Cansa precocemente. Podem ocorrer movimentos arrastados ocasionais.

$3=$ Prejudicado severamente. Hesitação freqüente ao iniciar os movimentos.

$4=$ Dificilmente realiza os movimentos.

25. Movimentos rápidos e alternados das mãos (pronação e supinação das mãos, verticalmente e horizontalmente, com a maior amplitude possível, com ambas as mãos simultaneamente)

$0=$ Normal.

$1=$ Um pouco lento e com redução na amplitude.

$2=$ Moderadamente comprometido. Cansa precocemente. Podem ocorrer movimentos arrastados ocasionais.

$3=$ Prejudicado severamente. Hesitação freqüente ao iniciar os movimentos.

$4=$ Dificilmente executa os movimentos.

\section{Agilidade das pernas}

$0=$ Normal.

$1=$ Um pouco lento e com redução na amplitude.

$2=$ Moderadamente comprometido. Cansa precocemente. Podem ocorrer movimentos arrastados ocasionais.

$3=$ Prejudicado severamente. Hesitação freqüente ao iniciar os movimentos.

$4=$ Dificilmente executa os movimentos. 


\section{Levantar da cadeira}

$0=$ Normal.

$1=$ Lento ou pode necessitar de esforço.

2 = Apóia-se nos braços da cadeira.

3 = Tende a cair para trás e pode tentar mais de uma vez, mas pode levantar-se sem auxílio.

4 = Incapaz de levantar-se sozinho.

\section{Postura}

$0=$ Normalmente ereto.

1 = Não completamente ereto, postura ligeiramente inclinada, pode ser considerado normal em caso de idade mais avançada.

2 = Postura moderadamente inclinada, definitivamente anormal, pode pender para algum lado.

$3=$ Postura seriamente inclinada com cifose, pode pender moderadamente para algum lado.

4 = Flexão intensa com extrema anormalidade na postura.

\section{Modo de andar}

$0=$ Normal.

1 = Anda lentamente, pode arrastar os pés em pequenos passos, não anda rápido e não salta.

$2=$ Anda com dificuldade sozinho ou com pequeno auxílio.

3 = Distúrbio grave na marcha, precisando de auxílio.

4 = Não conseguem andar, somente com assistência.

30. Estabilidade postural (Consiste de manobra súbita e com o paciente preparado, produzida empurrando os ombros do paciente ereto e com os olhos abertos e pernas afastadas)

$0=$ Normal.

1 = Retropulsão, mas se recupera sem auxílio.

2 = Ausência de resposta postural, cairá se não for auxiliado pelo examinador.

3 = Muito instável, perde o equilíbrio espontaneamente.

4 = Incapaz de ficar de pé sem auxílio.

31. Bradicinesia e hipocinesia (Combina lentidão, hesitação, diminuição no balanço dos braços, pequena amplitude e fraqueza dos movimentos em geral)

$0=$ Nenhuma.

1 = Mínima lentidão; simula um movimento proposital que pode ser normal para algumas pessoas. Pode ocorrer diminuição da amplitude.

2 = Leve grau de lentidão e fraqueza dos movimentos, definitivamente anormal. Certa diminuição da amplitude.

$3=$ Moderadamente lento, fraqueza e pequena amplitude dos movimentos.

$4=$ Lentidão acentuada, fraqueza e pequena amplitude dos movimentos. 
IV - COMPLICAÇÕES DO TRATAMENTO (na última semana)

\section{A. DISCINESIAS}

32. Duração: Em qual proporção do tempo em que você anda, as discinesias estão presentes?

$0=$ Nenhuma

$1=1-25 \%$ do dia

$2=26-50 \%$ do dia

$3=51-75 \%$ do dia

$4=76-100 \%$ do dia

33. Incapacidade: Qual é o grau de incapacidade ocasionado pelas discinesias?

0 = Não são incapacitantes

$1=$ Levemente incapacitantes

$2=$ Moderadamente incapacitantes

3 = Acentuadamente incapacitantes

4 = Completamente incapacitado

34. Discinesias dolorosas: Qual é a intensidade da dor provocada pelas discinesias?

$0=$ Indolor

$1=$ Leve

$2=$ Moderada

$3=$ Severa

$4=$ Grave

35. Presença de distonia no início da manhã

$0=$ Não

$1=\operatorname{Sim}$

\section{B. FLUTUAÇÕES CLÍNICAS}

36. Os períodos "off”(início dos sintomas) ${ }^{*}$ são previsíveis?

$0=$ Não

$1=\operatorname{Sim}$

37. Os períodos "off”(início dos sintomas)* são imprevisíveis?

$0=$ Não

$1=\operatorname{Sim}$

38. Os períodos “off"(sintomas da DP) ${ }^{*}$ iniciam subitamente, dentro de poucos segundos?

$0=$ Não

$1=\operatorname{Sim}$

* períodos "off"(início dos sintomas) - sem efeito do tratamento dopaminérgico (Winge et al., 2004) 
39. Em média qual proporção do dia você permanece com sintomas?

$0=$ Nenhum

$1=1-25 \%$ do dia

$2=26-50 \%$ do dia

$3=51-75 \%$ do dia

$4=76-100 \%$ do dia

C. OUTRAS COMPLICAÇÕES

40. Você apresenta anorexia, náuseas e vômitos?

$0=$ Não

$1=\operatorname{Sim}$

41. Você apresenta excesso de sono ou insônia?

$0=$ Não

$1=\operatorname{Sim}$

42. Você apresenta ortostase sintomática?

$0=$ Não

$1=\operatorname{Sim}$

TOTAL: 


\section{ANEXO C \\ QUESTIONÁRIO SIMPLIFICADO DA SOCIEDADE INTERNACIONAL DE CONTINÊNCIA PARA HOMENS}

Nós gostaríamos de saber quais são os seus sintomas urinários e agradecemos a sua cooperação em preencher este questionário.

Por favor, responda cada questão com base nos sintomas que você tem tido durante o último mês.

Algumas questões perguntam com que freqüência os sintomas aparecem. Responda de acordo com as seguintes instruções:

- Raramente $=$ menos de um terço das vezes

- Às vezes = entre um e dois terços das vezes

- Na maioria das vezes $=$ mais de dois terços das vezes

Marque com um X apenas uma alternativa para cada questão.

V1 - Quando você quer urinar, a urina demora em começar a sair?

0 ( ) nunca

1 ( ) raramente

2 ( ) às vezes

3 ( ) na maioria das vezes

4 ( ) sempre

V2 - Você tem que fazer força para continuar urinando?

0 ( ) nunca

1 ( ) raramente

2 ( ) às vezes

3 ( ) na maioria das vezes

4 ( ) sempre

V3 - Você diria que o seu jato de urina é:

0 ( ) normal

1 ( ) raramente fraco

2 ( ) às vezes fraco

3 ( ) na maioria das vezes fraco

4 ( ) sempre fraco 
V4 - Ao urinar, o seu jato de urina pára e recomeça mais de uma vez?

0 ( ) nunca

1 ( ) raramente

2 ( ) às vezes

3 ( ) na maioria das vezes

4 ( ) sempre

V5 - Com que freqüência você teve a sensação de não ter esvaziado completamente a bexiga após terminar de urinar?

0 ( ) nunca

1 ( ) raramente

2 ( ) às vezes

3 ( ) na maioria das vezes

4 ( ) sempre

I1 - Você tem que correr para o banheiro para urinar?

0 ( ) nunca

1 ( ) raramente

2 ( ) às vezes

3 ( ) na maioria das vezes

4 ( ) sempre

12 - Você chega a perder urina antes de chegar ao banheiro?

0 ( ) nunca

1 ( ) raramente

2 ( ) às vezes

3 ( ) na maioria das vezes

4 ( ) sempre

I3 - Você perde urina quando tosse ou espirra?

0 ( ) nunca

1 ( ) raramente

2 ( ) às vezes

3 ( ) na maioria das vezes

4 ( ) sempre

14 - Você perde urina sem causa aparente e sem vontade de urinar?

0 ( ) nunca

1 ( ) raramente

2 ( ) às vezes

3 ( ) na maioria das vezes

4 ( ) sempre 
15 - Você perde urina enquanto está dormindo?

0 ( ) nunca

1 ( ) raramente

2 ( ) às vezes

3 ( ) na maioria das vezes

4 ( ) sempre

16 - Com que freqüência você notou que suas roupas estavam um pouco molhadas logo após ter terminado de urinar e se vestido?

0 ( ) nunca

1 ( ) raramente

2 ( ) às vezes

3 ( ) na maioria das vezes

4 ( ) sempre

Freqüência:

Com que freqüência você urina durante o dia?

0 ( ) a cada 4 horas ou mais

1 ( ) a cada 3 horas

2 ( ) a cada 2 horas

3 ( ) de hora em hora

Noctúria:

Durante a noite, quantas vezes você se levanta para urinar, em média?

0 ( ) nenhuma

1 ( ) uma

2 ( ) duas

3 ( ) três

4 ( ) quatro ou mais

Qualidade de vida:

De um modo geral, quanto você acha que seus sintomas urinários interferem na sua vida?

0 ( ) Nem um pouco

1 ( ) Um pouco

2 ( ) Moderadamente

3 ( ) Bastante

Soma geral dos resultados: 


\section{ANEXO D}

\section{NOMOGRAMA DE SCHÄFER}

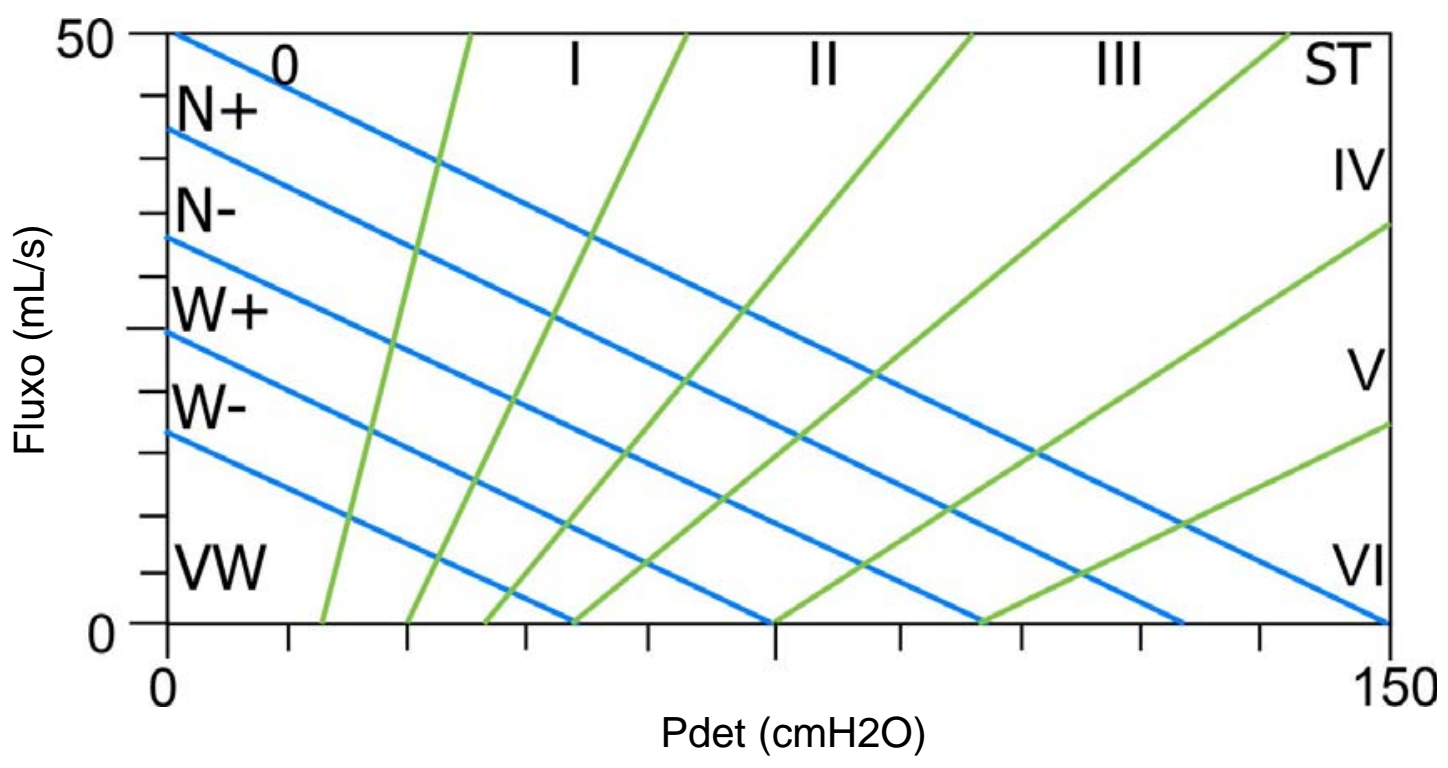

FONTE: Schäfer, 1990.

$\mathrm{N} \quad$ - Normal (normal)

ST - Strong (forte)

VW - Very weak (muito fraco)

W - Weak (fraco) 
8. REFERÊNCIAS 
Abrams P. Bladder outlet obstruction index, bladder contractility index and bladder voiding efficiency: three simple indices to define bladder voiding function. BJU Int. 1999;84(1):14-5.

Abrams P, Cardozo L, Fall M, Griffiths D, Rosier P, Ulmsten U, Van Kerrebroeck $V$, Wein $A$. The standardization of terminology of lower urinary tract function: report from the Standardization Sub-committee of the International Continence Society. Neurourol and Urodyn. 2002;21:167-78.

Alvord ECJr, Forno L, Skusske JA, Kauffman RJ, Rhodes JS, Goetowski CR. The pathology of Parkinsonism: a comparison of degenerations in cerebral cortex and brainstem. Adv Neurol. 1974;5:175-93.

Andersen JT, Bradley WE. Cystometric, sphincter and electromyographic abnormalities in Parkinson's disease. J Urol. 1976;116:75-8.

Araki I, Kitahara M, Oida T, Kuno S. Voiding dysfunction and Parkinson's disease: Urodynamic abnormalities and urinary symptoms. J Urol. 2000; 164:1640-643.

Araki I, Kuno S. Assessment of voiding dysfunction in Parkinson's disease by the international prostate symptom score. J Neurol Neurosurg Psychiatry. 2000;68:429-33.

Aranda B. Vesical sphincter disorders in Parkinson's disease. Rev Neurol (Paris). 1993;149(8-9):476-80. 
Baba Y, Putzke JD, Whaley NR, Wszolek ZK, Uitti RJ. Gender and the Parkinson's disease phenotype. Neurol. 2005;252(10):1201-205.

Baldereschi M, Di Carlo A, Rocca AW, Vanni P, Maggi S, Perissinotto E, Grigoletto F, Amaducci L, Inzitari D. Parkinson's disease and Parkinsonism in a longitudinal study: two fold higher incidence in men. Neurology. 2000; 55(9):1358-363.

Barry MJ, Fowler FJ, O'Leary MP, Bruskewitz RC, Holtgrewe HL, Mebust WK, Cockett AT. The American Urological Association Symptom Index for benign prostatic hyperplasia. The Measurement Committee of the American Urological Association. J Urol. 1992;148(5):1549-557.

Berger Y, Blaivas JG, De La Rocha ER, Salinas JM. Urodynamic findings in Parkinson's disease. J Urol. 1987;138(4):836-38.

Bonnet AM, Pichon J, Vidailhet M, Gouider-khouja N, Robain G, Perrigot M, Agid Y. Urinary disturbances in striatonigral degeneration and Parkinson's disease: clinical and urodynamic aspects. Mov Disord. 1997;12(4):509-13.

Bruschini H, Gomes CM, Figueiredo JA, Parra JAN, Arap S. Paraparesia espástica tropical e disfunção vesicoesfincteriana. J Bras Urol. 1996;22:78-80.

Campos-Sousa RN, Quagliato E, Silva BB, Carvalho Jr RM, Ribeiro SC, Carvalho DFM. Urinary symptoms in Parkinson's disease: prevalence and associated factors. Arq Neuropsiquiatr. 2003;61(2-B):359-63.

Conquy S, Zerbib M, Martinache PR, Thiounn N, Flam T, Debre B. Contribution of urodynamic examinations in the exploration of micturition disorders in patients with Parkinson's disease. J Urol (Paris). 1994;100(3):139-42. 
De Marinis M, Argenta G, Mele D, Carbone A, Baffigo G, Agnoli A. Evaluation of vesico-urethral and sweating function in disorders presenting with parkinsonism. Clin Auton Res. 1993;3(2):125-30.

Dias AE. Características fonoarticulatórias na doença de Parkinson de início na meia idade e tardio [tese]. São Paulo: Faculdade de Medicina, Universidade de São Paulo; 2006.

Dmochowski RR. Female voiding dysfunction and movement disorders. Int Urogynecol J Pelvic Floor Dysfunct. 1999;10(2):144-51.

Donovan JL, Peters TJ, Abrams P, Brookes ST, De La Rosette JJ, Schäfer W. Scoring the short form of ICSmaleSF questionnaire. International Continence Society. J Urol. 2000;164(6):1948-955.

Fahn S, Elton RI. Unified Parkinson's disease rating scale. In: Fahn S, Marsden CD, Calne DB, Goldstein M, editores. Recent development in Parkinson's disease. New York: Macmillan Health Care Information; 1987. v.2, p.153-64.

Fitzpatrick JM. The natural history of benign prostate hyperplasia. BJU Int. 2006; 97(Suppl 2):3-6.

Gomes CM, Trigo-Rocha FE, Arap MA, Arap S. Bladder outlet obstruction and urodynamic evaluation in patients with benign prostatic hyperplasia. Braz J Urol. 2001;27(6):575-88.

Gomes CM, Arap S, Trigo-Rocha FE. Voiding dysfunction and urodynamic abnormalities in elderly patients. Rev Hosp Clin Fac Med São Paulo. 2004a; 59(4):206-15. 
Gomes CM, Lopes RI, Sammour ZM, Barbosa ER, Haddad MS, Sallem FS. Lower urinary tract symptoms in patients with Parkinson's disease: Correlation with severity of neurological dysfunction based on different symptom scales. Mov Disord. 2004b; 19(Suppl 9): S 203. (Presented at 8th International Congress Parkinson's Disease and Movement Disorders, 2004b june 13-17; Rome, Italy. Abstract).

Gomes CM, Hisano M, Machado LR, Figueiredo JA, Lucon AM, Trigo-Rocha FE. Urological manifestations of chronic schistosomal myeloradiculopathy. BJU Int. 2005;96(6):853-56.

Hassler E, Krakau I, Haggarth L, Hassler E, Norlen L, Ekman P. Questioning questions about symptoms of benign prostatic hyperplasia. Fam Pract. 2001; 18(3):328-32.

Hattori N, Mizuno Y. Pathogenetic mechanisms of parkin in Parkinson's disease. Lancet. 2004;364(9435):722-24.

Hattori T, Yasuda K, Kita K, Hirayama K. Voiding dysfunction in Parkinson's disease. Jpn J Psychiatry Neurol. 1992;46(1):181-86.

Hobson P, Isla MW, Roberts S, Adhiyman V, Meara J. The risk of bladder and autonomic dysfunction in a community cohort of Parkinson's disease patients and normal controls. Parkinsonism Relat Disord. 2003;10(2):67-71.

Hoehn MM, Yahr MD. Parkinsonism: onset, progression and mortality. Neurology. 1967;17(5):427-42.

Hughes AJ, Daniel SE, Kilford L, Lees AJ. Accuracy of clinical diagnosis of idiopathic Parkinson's disease: a clinical-pathological study of 100 cases. J Neurol Neurosurg Psychiatry. 1992;55(3):181-84. 
Junemann KP, Melchior H. Disorders of bladder function in Parkinson's syndrome. Urologe A. 1990;29(4):170-75.

Khan Z, Starer P, Bhola A. Urinary incontinence in female Parkinson disease patients. Pitfalls of diagnosis. Urology. 1989;33(6):486-89.

Kuo HC. Videourodynamic characteristics and lower tract urinary symptoms in female bladder outlet obstruction. Urology. 2005;66(5):1005-9.

Lemack EG, Dewey RB Jr, Roehrborn CG, O`Suilleabhain PE, Zimmern EP. Questionnaire-based assessment of bladder dysfunction in patients with mild to moderate Parkinson's disease. Urology. 2000;56(2):250-54.

Lewin RJ, Dillard GV, Porter RW. Extrapyramidal inhibition of the urinary bladder. Brain Res. 1967;4(4):301-7.

Louis ED, Tang MX, Mayeux R. Parkinsonian signs in older people in a community-based study: risk of incident dementia. Arch. Neurol. 2004; 61(8):1273-276.

Magerkurth C, Schnitzer R, Braune S. Symptoms of autonomic failure in Parkinson's disease: prevalence and impact on daily life. Clin Auton Res. 2005;15(2):76-82.

McCrery RJ, Appell RA. Bladder outlet obstruction in women: iatrogenic, anatomic and neurogenic. Curr Urol Rep. 2006;7(5):363-69.

McGeer PL, McGeer EG. Inflammation and neurodegeneration in Parkinson's disease. Parkinsonism relat disord. 2004;10(Suppl1):3-7.

Pavlakis AJ, Siroky MB, Goldstein I, Krane RJ. Neurourologic findings in Parkinson's disease. J Urol. 1983;129(1):80-83. 
Resnick NM, Yalla SV. Detrusor hyperactivity with impaired contractile function. An unrecognized but common cause of incontinence in elderly patients. JAMA. 1987;257(22):3076-81.

Roehrborn CG, Boyle P, Gould AL, Waldstreicher J. Serum prostate-specific antigen as a predictor of prostate volume in men with benign prostatic hyperplasia. Urology. 1999;53(3):581-89.

Sacomani CA, Trigo-Rocha FE, Gomes CM, Greve JA, Barros TE, Arap S. Effect of the trauma mechanism on the bladder-sphincteric behavior after spinal cord injury. Spinal Cord. 2003;41(1):12-15.

Sakakibara R, Fowler CJ, Hattori T, Hussain IF, Swinn MJ, Uchiyama T, Yamanishi T. Pressure-flow study as an evaluating method of neurogenic urethral relaxation failure. J Auton Nerv Syst. 2000;80(1-2):85-8.

Sakakibara R, Hattori T, Uchiyama T, Yamanishi T. Videourodynamic and sphincter motor unit potential analyses in Parkinson's disease and multiple system atrophy. J Neurol Neurosurg Psychiatry. 2001a;71(5):600-6.

Sakakibara $R$, Shinotoh $H$, Uchiyama T, Sakuma $M$, Kashiwado $M$, Yoshiyama M, Hattori T. Questionnaire-based assessment of pelvic organ dysfunction in Parkinson's disease. Auton Neurosci. 2001b;92(1-2):76-85.

Samii A, Nutt JG, Ransom BR. Parkinson's disease. Lancet. 2004; 363(9423):1783-93.

Schapira AH. Disease modification in Parkinson's disease. Lancet Neurol. 2004;3(6):362-68.

Schäfer W, Rübben H, Noppeney R , Deutz FJ. Obstructed and unobstructed prostatic obstruction. World J Urol. 1989;6(4):198-203. 
Schäfer W. Principles and clinical application of advanced urodynamic analysis of voiding function. Urol Clin North Am. 1990;17(3):553-66.

Seif C, Herzog J, van der Horst C, Schrader B, Volkmann J, Deuschl G, Juenemann KP, Braun PM. Effect of subthalamic deep brain stimulation on the function of the urinary bladder. Ann Neurol. 2004;55(1):118-20.

Shimizu K, Yasukawa M, Yamamoto M, Hirao Y, Momose H, Kashiwai $H$, Kawata Y, Yamada K. Clinical findings of neurogenic bladder in patients with Parkinson's disease, multiple sclerosis and spinocerebellar degeneration. Hinyokika Kiyo. 1997;43(11):765-69.

Stocchi F, Carbone A, Inghilleri M, Monge A, Ruggieri S, Berardelli A, Manfredi M. Urodynamic and neurophysiological evaluation in Parkinson's disease and multiple system atrophy. J Neurol Neurosurg Psychiatry. 1997; 62:507-11.

Van Den Eeden SK, Tanner CM, Bernstein AL, Fross RD, Leimpeter A, Bloch DA, Nelson LM. Incidence of Parkinson's disease: variation by age, gender, and race / ethnicity. Am J Epidemiol. 2003;157:1015-22.

Winge K, Friberg L, Werdelin L, Nielsen KK, Stimpel H. Relationship between nigrostriatal dopaminergic degeneration, urinary symptoms, and bladder control in Parkinson's disease. Eur J Neurol. 2005;12(11):842-50.

Winge K, Skau AM, Stimpel H, Nielsen KK, Werdelin L.. Prevalence of bladder dysfunction in Parkinson's disease. Neurourol and Urodyn. 2006;25:116-22.

Winge K, Fowler CJ. Bladder dysfunction in Parkinsonism: mechanisms, prevalence, symptoms and management. Mov. Disorders. 2006;21(6):737-45. 


\section{APÊNDICES}




\title{
APÊNDICE I
}

\author{
HOSPITAL DAS CLÍNICAS \\ DA
}

FACULDADE DE MEDICINA DA UNIVERSIDADE DE SÃO PAULO

TERMO DE CONSENTIMENTO LIVRE E ESCLARECIDO

(Instruções para preenchimento no verso)

\section{I - DADOS DE IDENTIFICAÇÃO DO SUJEITO DA PESQUISA OU RESPONSÁVEL LEGAL}

1. NOME DO PACIENTE:
DOCUMENTO DE IDENTIDADE $\mathrm{N}^{\circ}$ SEXO : $M() F(\quad)$
DATA NASCIMENTO: $. / \ldots \ldots . . . . . . .$. $\mathrm{N}^{\mathrm{O}}$ APTO:
ENDEREÇO CIDADE

CEP: TELEFONE: DDD ..)

2.RESPONSÁVEL LEGAL

NATUREZA (grau de parentesco, tutor, curador etc.)

DOCUMENTO DE IDENTIDADE : SEXO: $M(\quad) F(\quad)$

DATA NASCI $. / \ldots . . . . . .$. No APTO:

BAIRRO: CEP: TELEFONE: DDD CIDADE: ..).

\section{II - DADOS SOBRE A PESQUISA CIENTÍFICA}

1. TÍTULO DO PROTOCOLO DE PESQUISA. Distúrbios miccionais em pacientes com doença de Parkinson: associação entre parâmetros clínicos e urodinâmicos

PESQUISADOR: Zein Mohamed Sammour. CARGO/FUNÇÃO: Médico pósgraduando. INSCRIÇÃO CONSELHO REGIONAL: No 59554. UNIDADE DO HCFMUSP: Instituto Central

3. AVALIAÇÃO DO RISCO DA PESQUISA:

SEM RISCO ( ) RISCO MÍNIMO ( $\mathrm{x}$ ) RISCO BAIXO ( ) RISCOMAIOR （ ) RISCO MÉDIO ( ) (probabilidade de que o indivíduo sofra algum dano como conseqüência imediata ou tardia do estudo)

4. DURAÇÃO DA PESQUISA: Início: 01-06-2003; Término: 31-06-2006. 


\section{III - REGISTRO DAS EXPLICAÇÕES DO PESQUISADOR AO PACIENTE OU SEU REPRESENTANTE LEGAL SOBRE A PESQUISA, CONSIGNANDO:}

1. Justificativa e os objetivos da pesquisa: Pacientes com doença de Parkinson podem apresentar sintomas relacionados ao controle da bexiga que incluem incontinência urinária, aumento da freqüência miccional, jato urinário fino e fraco e demora em iniciar a micção. Os sintomas podem ser causados somente pela doença de Parkinson ou podem ter outras causas agravantes, como doenças da próstata e da bexiga e infecções urinárias. O tratamento adequado desses sintomas requer um diagnóstico bem feito, que inclui exames laboratoriais, ultra-sonografia e exame urodinâmico. Além da determinação precisa do problema urinário, vamos avaliar a associação deste com a gravidade da doença de Parkinson visando prever o comportamento futuro desse problema. Precisamos, também, determinar o efeito que o problema urinário acarreta na qualidade de vida das pessoas doentes, para assim podermos instituir o tratamento mais adequado para cada caso.

2. Procedimentos que serão utilizados e propósitos, incluindo a identificação dos procedimentos que são experimentais: Sua participação nesta pesquisa não irá mudar nada em relação ao tratamento da sua doença de Parkinson ou dos sintomas urinários que você possa ter. Os exames que serão realizados serão os mesmos, independentemente da sua participação neste estudo. Apenas serão acrescentados questionários de sintomas (perguntas voltadas especificamente para avaliar seus sintomas urinários) que você será solicitado a preencher durante a avaliação médica. Serão efetuados exames de sangue, urina, ultra-som e exame urodinâmico.

3. Desconfortos e riscos esperados: Não há riscos ou possíveis seqüelas associados à participação neste estudo, isto é, os exames que serão realizados, embora tenham pequenos riscos associados, já são rotineiramente empregados nestes pacientes. Tais riscos são muito baixos e a participação ou não no estudo não afeta esses riscos, pois os critérios para a realização desses exames já são rotineiramente empregados e o estudo irá normatizar e acompanhar mais rigorosamente os pacientes.

4. Benefícios que poderão ser obtidos: Este estudo irá demonstrar a freqüência e a gravidade dos sintomas urinários nos pacientes com doença de Parkinson atendidos em nosso hospital. Mais do que isso, demonstrará se existe alguma correlação entre os problemas urinários e gravidade da doença de Parkinson, prejuízo na qualidade de vida e determinação das características dos sintomas miccionais que possam servir de base para 0 tratamento futuro de todos os doentes. 
5. Procedimentos alternativos que possam ser vantajosos para o indivíduo: Fui informado claramente de que os exames diagnósticos incluídos neste estudo são rotineiramente realizados na avaliação dos pacientes com doença de Parkinson que apresentem problemas urinários. Estou ciente de que este estudo não dispõe de qualquer exame novo ou que seja indicado de forma experimental, mas apenas normatiza e avalia detalhadamente os sintomas dos pacientes.

\section{IV - ESCLARECIMENTOS DADOS PELO PESQUISADOR SOBRE GARANTIAS DO SUJEITO DA PESQUISA:}

1. Acesso, a qualquer tempo, às informações sobre procedimentos, riscos e benefícios relacionados à pesquisa, inclusive para dirimir eventuais dúvidas. Estou ciente de que posso ter acesso a qualquer tempo às informações sobre os procedimentos, riscos e benefícios relacionados à pesquisa.

2. Liberdade de retirar seu consentimento a qualquer momento e de deixar de participar do estudo, sem que isso traga prejuízo à continuidade da assistência. Estou ciente de que não sou obrigado a participar deste estudo e de que a minha participação ou não no mesmo é uma decisão voluntária. Estou ciente também de que estou livre para voltar atrás na minha decisão de autorizar a participação neste estudo em qualquer instante.

3. Salvaguarda da confidencialidade, sigilo e privacidade. Estou ciente de que as informações a respeito dos meus sintomas de controle urinário são mantidas em sigilo e protegidas no arquivo de prontuários do Hospital das Clínicas. A liberação ou publicação de informações que identificam pacientes é proibida.

4. Disponibilidade de assistência no HCFMUSP, por eventuais danos à saúde, decorrentes da pesquisa. Estou ciente de que terei assistência médica garantida no HCFMUSP por eventuais danos à saúde decorrentes de minha participação neste estudo.

5. Viabilidade de indenização por eventuais danos à saúde decorrentes da pesquisa. 


\section{INFORMAÇÕES DE NOMES, ENDEREÇOS E TELEFONES DOS RESPONSÁVEIS PELO ACOMPANHAMENTO DA PESQUISA, PARA CONTATO EM CASO DE INTERCORRÊNCIAS CLÍNICAS E REAÇÕES ADVERSAS.}

Dr. Cristiano Mendes Gomes. End.: Rua Barata Ribeiro, 237, cj: 106 CEP: 01308-000 / FONE: 3120-2565 - São Paulo - SP. Divisão de Clínica Urológica - FMUSP - FONE: 3081-1091.

Dr. Zein Mohamed Sammour. End.: Rua Barão de Ladário, 540 CEP: 03010-000 / FONE: 6693-8215 / 8216 - São Paulo - SP. Divisão de Clínica Urológica - FMUSP - FONE: 3081-1091.

VI. OBSERVAÇÕES COMPLEMENTARES:

\section{VII - CONSENTIMENTO PÓS-ESCLARECIDO}

Declaro que, após convenientemente esclarecido pelo pesquisador e ter entendido o que me foi explicado, consinto em participar do presente Protocolo de Pesquisa

São Paulo,

de

de

assinatura do sujeito da pesquisa ou responsável legal

assinatura do pesquisador

(carimbo ou nome Legível) 


\section{APÊNDICE II}

\section{DIÁRIO MICCIONAL}

Nome:

Data:

Anotar neste diário por um período de 24 horas

Instruções:

1- Procure anotar o volume urinado corretamente. Você pode usar uma seringa de $20 \mathrm{~mL}$ ou outra maneira para medir, como você preferir.

2- Caso você apresente algum episódio de incontinência urinária, anoteo neste diário.

3- Preencha este diário em três dias diferentes, respeitando o intervalo de 7 (sete) dias entre as coletas dos dados.

4- Você deverá trazê-los devidamente preenchidos no dia da próxima avaliação.

5- O seguimento correto destas normas será muito importante para o sucesso do tratamento.

\begin{tabular}{|c|c|c|c|c|c|c|c|}
\hline $\begin{array}{l}\text { Horário } \\
\text { da } \\
\text { micção }\end{array}$ & $\begin{array}{l}\text { Volume } \\
\text { Urinado }\end{array}$ & $\begin{array}{c}\text { Desejo } \\
\text { incontrolável } \\
\text { de urinar }\end{array}$ & \begin{tabular}{|c|} 
Começou \\
e parou \\
de urinar \\
mais de \\
duas \\
vezes \\
\end{tabular} & \begin{tabular}{|c} 
Esvaziamento \\
incompleto \\
da bexiga
\end{tabular} & $\begin{array}{l}\text { Esforço } \\
\text { para } \\
\text { urinar }\end{array}$ & $\begin{array}{l}\text { Urinou } \\
\text { sem } \\
\text { força }\end{array}$ & Evacuações \\
\hline & & & & & & & \\
\hline & & & & & & & \\
\hline & & & & & & & \\
\hline & & & & & & & \\
\hline & & & & & & & \\
\hline & & & & & & & \\
\hline & & & & & & & \\
\hline & & & & & & & \\
\hline & & & & & & & \\
\hline & & & & & & & \\
\hline & & & & & & & \\
\hline & & & & & & & \\
\hline & & & & & & & \\
\hline & & & & & & & \\
\hline & & & & & & & \\
\hline & & & & & & & \\
\hline & & & & & & & \\
\hline
\end{tabular}

